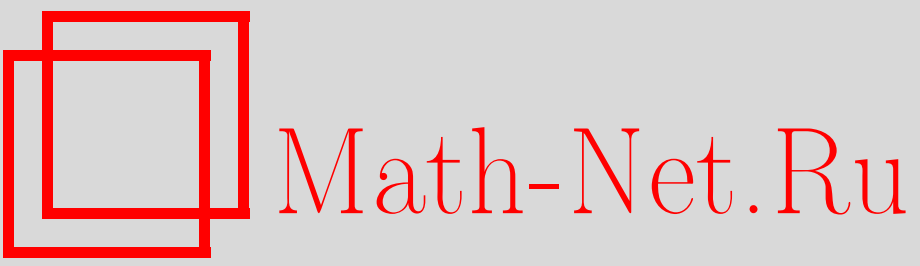

В. В. Федорчук, О некоторых вопросах топологической теории размерности, УМH, 2002, том 57, выпуск 2, 139-178

DOI: https://doi.org/10.4213/rm498

Использование Общероссийского математического портала Math-Net.Ru подразумевает, что вы прочитали и согласны с пользовательским соглашением

http://www . mathnet.ru/rus/agreement

Параметры загрузки:

IP : 54.80 .97 .219

26 апреля 2023 г., 16:03:23 


\title{
О НЕКОТОРЫХ ВОПРОСАХ ТОПОЛОГИЧЕСКОЙ ТЕОРИИ РАЗМЕРНОСТИ
}

\author{
В. В. ФЕДОРчУК
}

\begin{abstract}
Обзор посвящен вопросам теории размерности, связаным с работами Ю. М. Смирнова. Новые резултаты касаются размерности подмножеств многообразий. В предположении континуум-гипотезы строятся два бесконечномерных 4-многообразия. Первое из них является многообразием "без промежуточных размерностей”, т.е. всякое его замкнутое подмножество либо бесконечномерно, либо не более чем четырехмерно. Во втором многообразии размерности открытых его подмножеств принимают бесконечное число значений.

Библиографояи: 137 названий.
\end{abstract}

\section{СОДЕРЖАНИЕ}

Введение . . . . . . . . 139

$\S 1$. Некоторые вспомогательные результаты .......................... 142

$\S 2$. Конечномерные пространства ............................... 145

$\S 3$. Бесконечномерные пространства ............................... 153

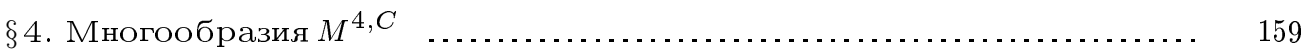

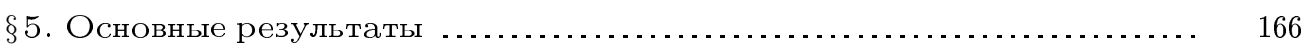

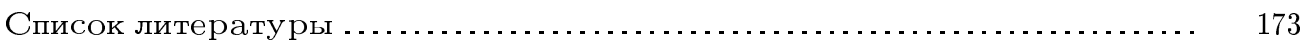

\section{Введение}

Затрагиваются вопросы теории размерности, в основном связанные с результатами Ю. М. Смирнова. Чисто обзорная часть статьи сосредоточена во втором и третьем параграфах. Ясно, что упомянуть все работы по освещаемым в обзоре разделам теории размерности невозможно. Например, Ю.М. Смирнов дал определение большой трансфинитной размерности Ind. Но из этого никак не вытекает, что в обзоре нужно и можно упомянуть все статьи, касаюшиеся этого инварианта. Стоит отметить, что не упомянуты и многие результаты самого Ю.М. Смирнова по разделам теории

Основные результаты статьи были получены во время пребывания автора в Университете г. Линчепинг (Швеция). Автор выражает искреннюю признательность за материалшную поддержку Королевской Шведской Академии Наук. Работа была поддержана также Российским фондом фундаменталшных исследований (грант № 00-01-00289). 
размерности, относяшимся к пространствам близости и равномерным пространствам, ретракциям, шэйпам, отображениям, пространствам с действием груп. Некоторую дополнительную информацию читатель может найти в монографии Энгелькинга [28], оказавшейся существенным подспорьем для автора при написании данной статьи, а также в обзорах [85] и [39].

Новые результаты статьи связаны с размерностью подмножеств многообразий. Известно, что всякое хаусдорфово паракомпактное многообразие метризуемо и, будучи связным, имеет счетную базу (см., например, [81]). Следовательно, всякое метризуемое (не обязательно связное) многообразие является дискретной суммой пространств со счетной базой. Поэтому из знаменитого тождества Урысона

$$
\text { ind } X=\operatorname{dim} X=\operatorname{Ind} X
$$

для пространств $X$ со счетной базой (см. [5]) вытекает, что

$$
\text { ind } A=\operatorname{dim} A=\operatorname{Ind} A \leqslant \operatorname{dim} M^{n}=n
$$

для всякого подмножества $A$ метризуемого $n$-многообразия $M^{n}$. Здесь ind и Ind соответственно малая и большая индуктивные размерности, a dim - лебегова размерность, определяемая посредством открытых покрытий.

В неметризуемом случае до построения $n$-многообразий $M^{n}$ с $\operatorname{dim} M^{n}>n$ речь могла идти лишш о соотношении между размерностями ind и Ind подмножеств многообразий $M^{n}$ размерности $\operatorname{dim} M^{n}=n$. В 1951 г. Ю.М. Смирнов [106] в предположении континуум-гипотезы доказал существование такого замкнутого подмножества $Y$ многообразия с краем $I \times L(L-$ “длинная прямая” П. С. Александрова, теоретико-множественное определение которой было дано еще Кантором [14]), что

$$
1=\operatorname{ind} Y<\operatorname{Ind} Y=\operatorname{dim}(I \times L)=2 \text {. }
$$

Из замкнутости множества $Y$ и равенства ind $Y=1$ вытекает его нигде не плотность в 2 -многообразии $I \times L$. Стоит отметить также, что многообразие с краем превращается в многообразие без края посредством склеивания со своим дубликатом по краю. При этом соотношения между размерностными инвариантами подмножеств нового многообразия совпадают с исходньми.

В 1992 г. автор [35] построил "наивный” (т.е. в аксиоматике ZFC) пример нормального счетно компактного 2-многообразия $M^{2}$, обладающего замкнутым нигде не плотным подмножеством $N$ размерности Ind $N=2=\operatorname{Ind} M^{2}$ и допускающего локально тривиальное расслоение на $L$.

Что касается размерности произведения многообразий, в [39] автором было доказано следуюшее обшее утверждение.

0.1. ТЕОРемА. Пусть $X$ - совериенно нормальное линделефово пространство, $Y$ - локально бикомпактное линейно упорядоченное пространство. Тогда если тождество Урысона (0.1) выполняется для $X$, то оно выполняется и для произведения $X \times Y$. При этом $\operatorname{dim}(X \times Y)=\operatorname{dim} X+\operatorname{dim} Y$.

Из теоремы 0.1 вытекает 
0.2. СЛЕДСТВИЕ. Если $M^{n}$ - метризуемое многообразие, $m о \operatorname{Ind}\left(M^{n} \times L\right)=$ $n+1$.

В связи с упомянутым вьше примером Смирнова возник следующий

0.3. Вопрос. Верно ли тождество Урысона для замкнутых подмножеств многообразий вида $M^{n} \times L$, где $M^{n}$ - компактное многообразие, при каких-нибудь теоретико-множественных предположениях?

А.В. Карасев [58] в предположении аксиомы Мартина (МА) и отрицания континуум-гипотезы $(\neg \mathrm{CH})$ дал положительньй ответ на этот вопрос. Пример Смирнова показывает, что в ZFC положительного ответа на вопрос 0.3 быть не может.

Формулировку аксиомы Мартина, вытекающей из континуум-гипотезы, можно найти, например, в [56]. Она эквивалентна следуюшему топологическому утверждению: компакт со свойством Суслина не может быть представлен в виде суммы менее чем континуума своих нигде не плотных подмножсеств. В [125] было доказано, что из непротиворечивости ZFC вытекает непротиворечивость ZFC+MA+ $\neg \mathrm{CH}$.

В 1992 г. В. В. Филиппов и автор [43] в предположении СН построили первые примеры многообразий с несовпадающими размерностями. А именно, для каждого $n \geqslant 3$ был построен пример нормального счетно компактного $n$-многообразия $M^{n}$ размерности $\operatorname{dim} M^{n}<\operatorname{Ind} M^{n}$. В 1993 г. автор [36] в предположении принципа Йенсена $\diamond$ (см. [57]), вытекающего из аксиомы конструктивности Геделя (см. [56]) и влекушего континуум-гипотезу, для всякого $n \geqslant 4$ построил дифференцируемое $n$-многообразие, все три основные размерности ind, dim, Ind которого попарно различны. Это многообразие было совершенно нормальным, счетно компактным и наследственно сепарабельным. В 1995 г. автор [37] построил аналогичный пример (кроме свойства счетной компактности), предполагая СН. Более точно, для любых $n \geqslant 4$ и $m>n$ было построено $n$-многообразие $M^{n, m}$ размерности

$$
\begin{aligned}
m-1 & \leqslant \operatorname{dim} M^{n, m} \leqslant m, \\
m+n-3 & \leqslant \operatorname{Ind} M^{n, m} \leqslant m+n-1 .
\end{aligned}
$$

С этого времени вопрос о размерности подмножеств многообразий стал значительно более многогранным. В частности, возник вопрос о промежуточных размерностях многообразий, т.е. вопрос о том, обязаны ли лебеговы размерности замкнутых подмножеств многообразия $M^{n}$ принимать все значения между $n$ и $\operatorname{dim} M^{n}$. Недавно А. Ч. Чигогидзе и автор [17] в предположении принципа $\diamond$ построили бесконечномерное 4-многообразие $M^{4}$, все конечномерные замкнутые подмножества которого не более чем 4-мерны. Там же построено бесконечномерное 4-многообразие, размерности открытых подмножеств которого принимают все значения $\geqslant 4$. Построенные в [17] многообразия дифференцируемы, счетно компактны, совершенно нормальны и наследственно сепарабельны.

В настоящей работе аналоги только что упомянутых результатов получены при более слабом, чем $\diamond$, предположении континуум-гипотезы. В бесконечномерном 4-многообразии $M_{1}^{4}$ из теоремы 5.5 нет замкнутых подмножеств конечной лебеговой размерности $n \geqslant 5$. Лебеговы размерности открытых подмножеств многообразия $M_{2}^{4}$ из 
теоремы 5.7 принимают бесконечное множество значений. Многообразия $M_{1}^{4}$ и $M_{2}^{4}$ обладают всеми дополнительными свойствами многообразий из [17], кроме счетной компактности. Стоит отметить, что без дополнительных теоретико-множественных предположений нельзя построить совершенно нормальные многообразия с такими размерностными свойствами. Это вытекает из известной теоремы М. Рудин [97] о том, что при $(\mathrm{MA}+\neg \mathrm{CH})$ всякое совершенно нормальное многообразие метризуемо.

Необходимые для дальнейшего вспомогательные результаты содержатся в $\S 1$. В $\S 4$ приводится основная конструкция. Для произвольного метризуемого компакта $C$ определяется факторное отображение $\varphi: B^{4} \rightarrow B_{C}^{4}$ замкнутого шара $B^{4}$ на компакт $B_{C}^{4}$, содержаший компакт $C$ в качестве нигде не плотного множества. При этом $\varphi: O^{4} \rightarrow$ $\varphi\left(O^{4}\right)$, где $O^{4} \subset B^{4}$ - открытый шар, является гомеоморфизмом на всюду плотное открытое подмножество компакта $B_{C}^{4}$. После этого трансфинитной рекурсией строится непрерьвный обратньй спектр $S^{\varphi}=S^{\varphi, C}$ длины $\omega_{1}$, состояший из компактов $B_{C}^{4}$ и почти гомеоморфизмов в качестве связывающих проекщий. Основньм объектом исследования в статье служит 4-многообразие $M^{4, C}$, являющееся всюду плотным открытым подмножеством бикомпакта $X=\lim S^{\varphi}$. Вся конструкция и основные топологические свойства многообразия $M^{4, C}$ базируются на континуум-гипотезе.

Прототипы спектров $S^{\varphi}$ в частном случае $C=I^{m}$ появились в работах [36] и [37] в предположениях $\diamond$ и $\mathrm{CH}$ соответственно. В общем случае спектр $S^{\varphi}$ при $\diamond$ был определен в [38].

Главные результаты статьи (теоремы 5.5 и 5.7 ) базируются на теореме 5.1, которая с учетом замечания 5.8 распространяет формулу (0.2) на произвольные замкнутые подмножества многообразия $M^{4, C}$. В статье приводится цельй ряд вопросов и комментарии к ним.

\section{§1. Некоторые вспомогательные результаты}

Топологическим $n$-многообразием назьвается связное хаусдорфово пространство, локально гомеоморфное $n$-мерному евклидову пространству $\mathbb{R}^{n}$. Определение дифференцируемого (или гладкого) $n$-многообразия можно найти, например, в книге [94]. Основные понятия и факты общей топологии и теории размерности читатель может найти в книгах [5], [42] и [28].

Все пространства предполагаются тихоновскими, все отображения - непрерьвными. Хаусдорфовы бикомпактные пространства называются компактами. Замыкание (соответственно внутренность) подмножества $Y$ топологического пространства $X$ обозначается через $\mathrm{Cl}_{X}(Y)\left(\right.$ соответственно $\left.\operatorname{Int}_{X}(Y)\right)$ или просто через $\mathrm{Cl}(Y)(\operatorname{Int}(Y))$. Когда мы говорим, что топологическое пространство $X n$-мерно, мы имеем в виду, что $\operatorname{dim} X=n$. Пространство $X$ называется индуктивно $n$-мернылм, если ind $X=n$.

Через $|A|$ мы обозначаем мошность множества $A$. Порядковое число отождествляется с множеством меньших порядковых чисел, кардинальное число - это наименьшее порядковое число данной мошности. В соответствии с этим бесконечное кардинальное число обозначается символом $\omega_{\alpha}$, где $\alpha$ - порядковое число. Так, $\omega_{0}-$ наименьшее бесконечное кардинальное число, $\omega_{1}$ - наименьшее несчетное кардинальное число и т. д. При этом приходится мириться с тем, что множество всех неотрицательных целых чисел обозначается двумя способами: $\omega$ как множество порядковых чисел и $\omega_{0}$ как кардинальное число. 
Подмножество $F$ топологического пространства $X$ назьвается нуль-мно жеством, если существует такая функция $\varphi: X \rightarrow \mathbb{R}$, что $F=\varphi^{-1}(0)$. Дополнения до нуль-множеств назьваются конуль-множествами или функиионально открытыми множествами. Из леммы Урысона вытекает, что в нормальных пространствах функционально открытые множества - это в точности открытые $F_{\sigma}$-множества.

Пусть $X$ - топологическое пространство, и пусть $A$ и $B$ - его замкнутые дизъюнктные подмножества. Замкнутое множество $C \subset X$ назьвается перегородкой в пространстве $X$ между множествами $A$ и $B$, если $X \backslash C$ есть объединение непересекающихся окрестностей $O A$ и $O B$.

Одним из фундаментальных фактов теории размерности является теорема о перегородках (теорема 1.1). Эту теорему доказали Отто и Эйленберг [26] для пространств со счетной базой и Хеммингсен [47] в общем случае.

1.1. Теорема. Пусть $X$ - нормальное пространство. Тогда $\operatorname{dim} X \geqslant n$ в том и только том случае, когда существуют парьи $\left(A_{i}, B_{i}\right), 1 \leqslant i \leqslant n$, непересекающихся замкнутых подмножеств пространства $X$ такие, что $\bigcap\left\{C_{i}: 1 \leqslant i \leqslant n\right\}$ $\neq \varnothing$ для любых перегородок $C_{i}$ в пространстве $X$ межсду множествами $A_{i}$ $u B_{i}$.

Из этой теоремы вытекает

1.2. ПРЕДЛОЖЕНИЕ. Если $\operatorname{dim} X \geqslant n$ для нормального пространства $X$, то сущ,ествуют такие пары $\left(A_{i}, B_{i}\right), 1 \leqslant i \leqslant n-1$, непересекающихся замкнутых подмножсеств пространства $X$, что $\operatorname{dim}\left(\bigcap\left\{C_{i}: 1 \leqslant i \leqslant n-1\right\}\right) \geqslant 1$ для любьх перегородок $C_{i}$ в пространстве $X$ межсду множсествами $A_{i} u B_{i}$.

Пусть $f: X \rightarrow Y$ - отображение на $Y$. Тогда для $A \subset X$ мы полагаем

$$
f^{\#} A=\left\{y \in Y: f^{-1} y \subset A\right\}=Y \backslash f(X \backslash A) .
$$

1.3. ПРЕДЛОЖЕНИЕ. Пусть $f: X \rightarrow Y$-монотонное, замкнутое отображение на $Y$, и пусть $A$ и $B$ - дизбюнктные замкнутые подмножества пространства $Y$. Тогда для любой перегородки $C$ в пространстве $X$ междуумножествами $f^{-1} A$ и $f^{-1} B$ множество $f(C)$ является перегородкой в $Y$ межсду $A$ и $B$.

Доказательство этого утверждения, являющегося математическим фольклором, основано на том, что при замкнутом отображении $f: X \rightarrow Y$ мальй образ $f$ \# $U$ произвольного открытого множества $U \subset X$ открыт. Поэтому если $X \backslash C=O f^{-1} A \cup$ $O f^{-1} B$ есть дизъюнктная сумма открытых множеств, то $Y \backslash f(C)=f^{\#}\left(O f^{-1} A\right) \cup$ $f^{\#}\left(O f^{-1} B\right)$ также является дизъюнктной суммой открытых множеств.

Говорят, что замкнутое множество $C \subset X$ разбивает пространство $X$, если оно является перегородкой в $X$ между некоторыми двумя различными точками из $X$. Компакт $X$ назьвается канторовым $n$-многообразием, если $\operatorname{dim} X=n$ и $X$ нельзя разбить множеством размерности $\leqslant n-2$.

1.4. ТЕОремА. Всякий n-мерный компакт содержит канторово п-многообразие.

Эта теорема для метризуемых компактов была независимо получена Гуревичем и Менгером [50] и Тумаркиным [127], в обшем случае - П. С. Александровьп [3]. 
1.5. ТЕОРема ДАУКеРА [21]. Пусть $F$ - замкнутое подмножество нормального пространства $X$. Предположим, что $\operatorname{dim} F \leqslant n u \operatorname{dim} A \leqslant n$ для всякого замкнутого в $X$ множества $A$, лежащего в $X \backslash F$. Тогда $\operatorname{dim} X \leqslant n$.

1.6. ПРЕДЛОЖЕНИЕ. Пусть $f: X \rightarrow Y$ - отображение между компактами. Если $Y$ и все слои $f^{-1}$ у удовлетворяют первой аксиоме счетности, то $X$ также удовлетворяет первой аксиоме счетности.

Это - также математический фольклор, основанньй на совпадении характера и псевдохарактера в компактах. И следуюшее утверждение является математическим фольклором.

1.7. ПРЕДЛОЖЕНИЕ. Пусть $X-$ пространство, удовлетворяющее первой аксиоме счетности, а $Y$ - его счетное компактное подмножество. Тогда $Y$ является $G_{\delta}$-множеством.

Доказательство можно провести индукцией по производной высоте множества $Y$, используя то, что при добавлении к замкнутому $G_{\delta}$-множеству счетного числа изолированных точек счетного характера вновь получается $G_{\delta}$-множество.

Напомним, что отображение $f: X \rightarrow Y$ назьвается почти гомеоморфизмом, если для всякого открытого покрытия $u$ пространства $Y$ сушествует гомеоморфизм $g: X \rightarrow$ $Y$, который $u$-близок к отображению $f$, т.е. для любого $x \in X$ сушествует такой элемент $U \in u$, что $f(x), g(x) \in U$.

1.8. АППРОКСИМАШИОННАЯ ЛЕММА БРАУНА [13]. Пусть $\left\{X_{i}, f_{i-1}^{i}\right\}-$ обратная последовательность, состоящая из метризуемых компактов и почти гомеоморфизмов, и пусть $X=\lim \left\{X_{i}, f_{i-1}^{i}\right\}$. Тогда сквозная проекиия $f_{0}: X \rightarrow X_{0}$ также является почти гомеоморфизмом.

1.9. ЗАмечАниЕ. Что касается этого утверждения, в работе [13] доказано лишш то, что компакты $X$ и $X_{0}$ гомеоморфны. Но слегка модифищируя доказательство леммы из [13], получаем доказательство леммы 1.8 .

Пусть

$$
S=\left\{X_{\alpha}, \pi_{\beta}^{\alpha}, \beta \leqslant \alpha<\gamma\right\} \equiv\left\{X_{\alpha}, \pi_{\beta}^{\alpha}, \gamma\right\}
$$

- обратньй спектр (топологических пространств) множеств, заданньй над начальным интервалом $[0, \gamma)$ семейства всех порядковых чисел, и пусть $\alpha<\gamma-$ предельное порядковое число. Через $S \mid \alpha$ обозначим ограничение спектра $S$ на множество $\alpha$, т.е.

$$
S \mid \alpha=\left\{X_{\beta}, \pi_{\beta^{\prime}}^{\beta}\right\} .
$$

Положим $X^{\alpha}=\lim (S \mid \alpha)$ и обозначим сквозные проекции $X^{\alpha} \rightarrow X_{\beta}$ через $p_{\beta}^{\alpha}$. Из определения предела обратного спектра вытекает сушествование единственного (непрерьвного) отображения $p_{\alpha}: X_{\alpha} \rightarrow X^{\alpha}$, удовлетворяющего условию $p_{\beta}^{\alpha} \circ p_{\alpha}=\pi_{\beta}^{\alpha}$ для всех $\beta<\alpha$. Напомним, что спектр $S$ назьвается непрерывныл, если отображение $p_{\alpha}$ является биекцией (гомеоморфизмом) для всякого предельного порядкового числа $\alpha<\gamma$.

1.10. ЗАМЕЧАНИЕ. Ясно, что для определения непрерывного обратного спектра $S$ достаточно задать все его короткие проекции $\pi_{\alpha}^{\alpha+1}$.

Трансфинитной индукцией по длине $\gamma$ спектра $S=\left\{X_{\alpha}, \pi_{\beta}^{\alpha}, \gamma\right\}$ доказьвается следуюшее обобщение леммы 1.8 . 
1.11. ЛЕмМА БрАУНА. Пусть $S=\left\{X_{\alpha}, \pi_{\beta}^{\alpha}, \gamma\right\}$ - счетный непрерывный обратный спектр, состоящий из метризуемых компактов, все короткие проекции $\pi_{\alpha}^{\alpha+1}$ которого являются почти гомеоморфизмами. Тогда для любого $\beta<\gamma$ сквозная проекиия $\pi_{\beta}: \lim S \rightarrow X_{\beta}$ также является почти гомеоморфизмом.

При доказательстве леммы 1.11, кроме леммы 1.8, используется следуюшее достаточно очевидное утверждение.

1.12. ПРЕДЛОЖЕНИЕ. Композичия двух почти гомеоморфизмов является почти гомеоморфизмом.

1.13. ТеОрема КоЗЛОВСКОГО И ЗЕнора [62]. Если дифференцируемое многообразие $M^{n}$ имеет такой атлас $\left\{\left(U_{i}, \varphi_{i}\right): i \in \omega\right\}$, что $U_{i} \subset U_{i+1} u \varphi_{i}\left(U_{i}\right)=\mathbb{R}^{n}$ для всех $i \in \omega$, то $M^{n}$ диффеоморфно $\mathbb{R}^{n}$.

1.14. ТЕОРема (см. [81; следствие 2.18]). Всякое сепарабельное совериенно нормальное многообразие наследственно сепарабельно.

Пусть $A$ - замкнутое подмножество топологического пространства $X$, и пусть $\varphi: A \rightarrow B-$ факторное отображение на некоторое пространство $B$. Обозначим через $X \varphi$ факторпространство пространства $X$ относительно разбиения, элементы которого суть слои $\varphi^{-1} b$ отображения $\varphi$ и одноточечные множества из $X \backslash A$. Факторное отображение $X \rightarrow X_{\varphi}$ обозначим через $p_{\varphi}$.

1.15. ЛЕмма (основная лемма из [36]). Пусть $B^{n}-$ замкнутый $n$-шар, $n \geqslant 2$, пусть $S^{n-1}$ - его граница и $O^{n}=B^{n} \backslash S^{n-1}$. Пусть $A$ - замкнутое подмножество сферы $S^{n-1}$, а $\varphi: A \rightarrow B$ - непрерывное отображение на некоторый компакт $B$. Предположим, что для некоторой точки $b_{0} \in B$ множество $L=\varphi^{-1} b_{0}$ связно и нигде не плотно в $S^{n-1}$. Предположим, далее, что $h: L \times[0,1] \rightarrow B^{n}$ является вложением, удовлетворяющим следующим условияM:

1) $h(l, 0)=l$ для любого $l \in L$;

2) $h(L \times(0,1]) \subset O^{n}$.

Тогда для произвольного счетного семейства $\mathscr{C}=\left\{C_{i}: i \in \omega\right\}$ таких счетных множеств $C_{i} \subset O^{n}=B_{\varphi}^{n} \backslash S_{\varphi}^{n-1}$, что $y=p_{\varphi}\left(\varphi^{-1} b_{0}\right)$ есть предельная точка множества $C_{i}$ для любого $i$, существует непрерывное отображсение $g=$ $g_{(y, \mathscr{C})}: B_{\varphi}^{n} \rightarrow B_{\varphi}^{n}$ со следующими свойствами:

3) g есть почти гомеоморфизм;

4) $g$ имеет только один нетривиальньй слой $g^{-1} y=p_{\varphi} h(L \times[0,1]) \equiv K$;

5) $g \mid S_{\varphi}^{n-1}=\mathrm{id}$

6) $g: O^{n} \backslash K \rightarrow O^{n}$ есть диффеоморфизм;

7) $K \subset \mathrm{Cl}\left(g^{-1}\left(C_{i}\right)\right)$ для любого $i$.

\section{§. Конечномерные пространства}

В 1911 г. Брауэр [11] доказал, что кубы $I^{m}$ и $I^{n}$ не гомеоморфны при $m \neq n$. При этом он показал, что единичньй куб $I^{n}$ при $\varepsilon<\frac{1}{2}$ нельзя отобразить посредством $\varepsilon$-сдвига ни на какое лежашее в кубе $I^{n}$ нигде не плотное в нем множество. Тогда же Лебег [64] также предпринял попытку доказательства негомеоморфности кубов 
$I^{m}$ и $I^{n}$. Но его доказательство содержало пробелы, заполненные им в 1921 г. [65]. Но бессмертньй вклад Лебега в будущую теорию размерности состоял в том, что в упомянутой работе 1911 г. он дал определение (применительно к кубу $I^{n}$ ) основного в настоящее время размерностного инварианта dim, справедливо назьваемого также лебеговой размерностью.

В 1913 г. Брауэр [12] дал индуктивное определение размерности метризуемых компактов, основываясь на идеях Пуанкаре ([86], [87]). Свой размерностный инвариант (прототип большой индуктивной размерности) Брауэр назвал Dimensiongrad (мы будем обозначать его символом $\mathrm{Dg}$ ). Определение Брауэра основано на понятии разpeза. Скажем, что замкнутое подмножество $T$ пространства $X$ разрезает $X$ между непересекаюшимися замкну тыми множествами $A$ и $B$ этого пространства (или является разрезом в $X$ между $A$ и $B$ ), если всякий континуум (связный компакт) $C \subset X$, пересекаюшийся с $A$ и $B$, пересекается также и с $T$. Брауэр определяет нульмерные в смысле Dg пространства как непустые метризуемые компакты, не содержашие неодноточечных континуумов. Далее, $\operatorname{Dg} X \leqslant n$ тогда и только тогда, когда для всякой пары $A$ и $B$ непересекаюшихся замкнутых подмножеств пространства $X$ сушествует разрез $T$ в $X$ между $A$ и $B$ размерности $\operatorname{Dg} T \leqslant n-1$.

Определение инварианта $\mathrm{Dg}$ не совпадает с определением большой индуктивной размерности Ind для метризуемых компактов, поскольку не всякий разрез является перегородкой. Так, например, в любом невырожденном наследственно неразложимом континууме (т.е. таком континууме, что никакой его подконтинуум не является объединением двух своих собственных подконтинуумов) между любыми двумя его непересекаюшимися подконтинуумами существует разрез, состоящий из одной точки. Но в полных локально связных метрических пространствах (в частности, в пеановских континуумах) понятия разреза и перегородки совпадают. Поэтому долгое время считалось, что определение Брауэра совпадает с определением размерности Ind для класса всех полных локально связных метрических пространств. Ввиду "очевидности" этого факта попыток его строгого доказательства не предпринималось.

В 1951 г. Бинг [8] доказал, что между любыми двумя замкнутыми непересекаюшимися подмножествами гильбертова куба существует перегородка, каждая компонента связности которой является наследственно неразложимым континуумом. Отсюда вытекает вьполнение этого же свойства в любом метрическом компакте. Поэтому элементарной индукцией нельзя доказать равенство $\operatorname{Dg} I^{n}=\operatorname{Ind} I^{n}$. Тем не менее, это равенство оказалось справедливым. Более того, имеет место

\section{1. Теоремa [40]. $\operatorname{Dg} X=\operatorname{dim} X$ для всякого метризуемого компакта.}

Доказательство этой теоремы основьвается на трех фундаментальных результатах теории размерности: теореме счетной суммы для замкнутых слагаемых, теореме Тумаркина о $G_{\delta}$-оболочке той же размерности и аддиционной теореме. Эти факты не могли быть даже сформулированы ко времени написания Брауэром своей статьи [12]. Поэтому Лебег был все-таки первым, кто доказал топологическую $n$-мерность $n$-мерного куба.

Что касается локально связных полных метрических пространств, высказывание о том, что функция Dg является хорошим размерностным инвариантом для этого класса пространств, оказалось абсолютно неверным. 
2.2. Теорема [41]. Для всякого $n \geqslant 2$ существует локально связное полное сепарабельное метрическое пространство $X_{n}$ размерности

$$
\operatorname{Dg} X_{n}=1<n=\operatorname{dim} X_{n} .
$$

Неудовлетворительность брауэровского подхода к определению размерности (даже для метрических компактов) можно проиллюстрировать еще одним способом. Естественным образом определяется малый индуктивный инвариант $\mathrm{dg}$ (dimensiongrad). Оказывается [40], имеет место

2.3. ТЕОРемА. $\operatorname{dg} X \leqslant 2$ для всякого метризуемого компакта $X$.

Упомянутые вьше теорема счетной суммы для замкнутых слагаемых и аддиционная теорема были доказаны П. С. Урысоном. Последняя была им сфформулирована в виде неравенства

$$
\operatorname{ind}(X \cup Y) \leqslant \operatorname{ind} X+\operatorname{ind} Y+1
$$

и доказана для подмножеств метрическох компактов. В силу тождества Урысона, неравенство (2.1) можно записать еще двумя другими способами. А именно:

$$
\begin{aligned}
& \operatorname{Ind}(X \cup Y) \leqslant \operatorname{Ind} X+\operatorname{Ind} Y+1, \\
& \operatorname{dim}(X \cup Y) \leqslant \operatorname{dim} X+\operatorname{dim} Y+1 .
\end{aligned}
$$

Доказательство Урысона неравенства (2.1) непосредственно переносится на наследственно нормальные пространства. Неравенства (2.2) и (2.3) для наследственно нормальных пространств были доказаны Ю.М. Смирновьм [106]. А.В. Зарелуа [135] распространил неравенство (2.3) на нормальные пространства.

Много сил затратили специалисты по теории размерности, пытаясь распространить тождество Урысона за пределы сепарабельных метризуемых пространств. Эта проблема естественно расчленилась на несколько задач о соотношении между основными размерностными инвариантами dim, ind, Ind в различных классах пространств. Достаточно полную историю этого вопроса можно найти в обзоре [39]. Здесь же мы отметим лиш те аспекты этой проблемы, которые непосредственно связаны с результатами Ю.М. Смирнова. Одной из составляюших тождества Урысона является неравенство

$$
\operatorname{dim} X \leqslant \operatorname{ind} X
$$

В 1940 г. П. С. Александров [2] доказал это неравенство для компактов. В 1950 г. Морита [72] доказал неравенство (2.4) для финально компактных, а затем и для сильно паракомпактных пространств [73]. Независимо от Мориты неравенство (2.4) для финально компактных пространств получил Ю. М. Смирнов [106]. В 1961 г. А. В. Зарелуа (см. [133], [134]) распространил неравенство (2.4) на вполне паракомпактные пространства. Напомним, что топологическое пространство называется вполне паракомпактным, если в любое его открытое покрытие можно вписать открытое покрытие, распадающееся в счетную сумму систем, каждую из которых можно продолжить до звездно конечного открытого покрытия всего пространства. В частности, метризуемые вполне паракомпактные пространства - это в точности пространства с $\sigma$-звездно конечной базой. Такие пространства назьваются сильно метризуемыми. Десятью годами ранее Катетов ([59], [60]) доказал свою знаменитую теорему. 
2.4. ТЕОРемА. Для метрического пространства $X$ следующие условия равносильныр:

(1) $\operatorname{dim} X=n$;

(2) Ind $X=n$;

(3) $X=X_{0} \cup X_{1} \cup \cdots \cup X_{n}$, zдe $\operatorname{dim} X_{i}=0$.

Из этой теоремы и упомянутой вьше теоремы Зарелуа вытекает

2.5. ТеоремА. Для сильно метризуемого пространства $X$ имеет место тождество Урысона

$$
\operatorname{dim} X=\operatorname{ind} X=\operatorname{Ind} X \text {. }
$$

Построенный в 1962 г. Роем ([95], [96]) пример полного метрического пространства $R$ размерности

$$
0=\operatorname{ind} R<\operatorname{dim} R=1
$$

показал, что распространить утверждение теоремы 2.5 на все метрические пространства нельзя.

2.6. ЗАмечАниЕ. В 1951 г. Ю.М. Смирнов [106] построил первый пример нормального пространства с несовпадаюшими индуктивными размерностями. Его пространство $S$ построено без дополнительных теоретико-множественных предположений. Оно является подмножеством произведения $I \times L\left(2^{\omega_{0}}\right)$, где $L\left(2^{\omega_{0}}\right)$ - "трансфинитная прямая" длины континуум, получаюшаяся, как и “длинная прямая", заполнением каждого скачка множества порядковых чисел $\alpha<2^{\omega_{0}}$ экземпляром интервала $(0,1)$ числовой прямой $\mathbb{R}$. В предположении континуум-гипотезы "трансфинитная прямая" $L\left(2^{\omega_{0}}\right)$ преврашается в “длинную прямую” $L=L\left(\omega_{1}\right)$.

Важньм разделом теории размерности является проблема монотонности размерности, т.е. вопрос о выполнимости неравенства

$$
d X_{0} \leqslant d X
$$

для подпространства $X_{0}$ пространства $X$ и размерностного инварианта $d$. Размерность ind монотонна по любым подмножествам. Из теоремы суммы вытекает, что размерность $\operatorname{dim}$ монотонна по $F_{\sigma}$-подмножествам. Поэтому $\operatorname{dim}$ монотонна в классе всех совершенно нормальных пространств. Это утверждение и аналогичное утверждение для размерности Ind доказал Чех [15]. Ю. М. Смирнов [107] доказал, что если подпространство $X_{0}$ нормально расположено в нормальном пространстве $X$, то

$$
\operatorname{dim} X_{0} \leqslant \operatorname{dim} X
$$

При этом подпространство $X_{0}$ нормального пространства $X$ назьвается нормально расположенным в $X$, если в любой окрестности множества $X_{0}$ найдется множество $X_{1} \supset X_{0}$ типа $F_{\sigma}$ в $X$. Поскольку всякое финально компактное пространство нормально расположено в любом объемлющем его пространстве, имеет место 
2.7. Теорема [107]. Неравенство (2.6) верно для любого финально компактного подпространства $X_{0}$ нормального пространства $X$.

Морита [74] доказал неравенство (2.6) для сильно паракомпактного $X_{0}$, а В. П. Золотарев [137] - для вполне паракомпактного $X_{0}$. Даукер [20] доказал монотонность размерности Ind в классе вполне нормальных пространств. При этом, нормальное пространство $X$ называется вполне нормальныцм, если всякое его открытое подмножество $U$ имеет локально конечное покрытие, состоящее из функционально открытых в $X$ множеств. Энгелькинг [28] распространил теорему Даукера на класс сильно наследственно нормальных пространств, определение которых получается из определения вполне нормальных пространств заменой локально конечных покрытий на точечно конечные.

В 1955 г. Даукер [21] построил первый пример компакта $X$, в котором размерности $\operatorname{dim}$ и Ind могли повьшаться при переходе к нормальному подпространству $X_{0}$. Его компакт $X$ нульмерен, a Ind $X_{0}=1$ (следовательно, $\left.\operatorname{dim} X_{0}=1\right)$. В 1958 г. Ю. М. Смирнов [113] построил нульмерньй компакт $Y$ и его бесконечномерное подпространство $Y_{0}$, большая индуктивная размерность которого не определена. При этом $Y_{0}$ было первым примером нормального индуктивно-нульмерного пространства, лебегова размерность которого бесконечна.

В.В. Филиппов дал отрицательное решение [44] проблем Чеха и Даукера о монотонности размерностей dim и Ind. Им в предположении существования суслинского контину ума было построено нульмерное наследственно нормальное пространство, содержащее подпространства всех конечных в смысле и dim и Ind размерностей.

Первые наследственно нормальные компакты, в которых размерности dim и Ind не монотонны, в предположении принципа Йенсена $\diamond$ построил автор ([31], [32]). Были построены как компакты, в которых размерности dim и Ind одновременно не монотонны, так и компакты, в которых $\operatorname{dim}$ монотонна, a Ind нет. При этом размерность повьшалась при переходе к совершенно нормальному счетно-компактному подпространству. Построить такой пример без дополнительных теоретико-множественных предположений нельзя, поскольку, как показал Вейс [132] в предположении аксиомы Мартина и отрицания континуум-гипотезы, всякое совершенно нормальное счетно-компактное пространство компактно. Затем Осташевский [83], также предполагая $\diamond$ построил нульмерный компакт, который содержит совершенно нормальные счетно-компактные подпространства $\Omega_{n}$ размерности $\operatorname{dim} \Omega_{n}=\operatorname{Ind} \Omega_{n}=n$.

Первые ZFC-примеры наследственно нормальных пространств, в которых размерности $\operatorname{dim}$ и Ind не монотонны, построили Р. Поль и Э. Поль ([92], [91]). Э. Поль в предположении $\mathrm{CH}$ построила [88] нульмерньй наследственно нормальньй компакт с подпространствами положительной размерности. Автор, также предполагая континуум-гипотезу, построил [33] нульмерньй наследственно нормальньй компакт, содержаший подпространства $U_{m n}, 1 \leqslant m \leqslant n$, размерности $\operatorname{dim} U_{m n}=m, \operatorname{Ind} U_{m n}=n$. Нерешенным остается основной вопрос в этой тематике.

2.8. Вопрос. Можно ли без дополнительных теоретико-множественных предположений построить наследственно нормальньй компакт, в котором размерности $\mathrm{dim}$ и Ind не монотонны? 
Большой раздел теории размерности связан с компактификациями. Равенства

$$
\operatorname{dim} X=\operatorname{dim} \beta X, \quad \text { Ind } X=\operatorname{Ind} \beta X
$$

для нормальных пространств $X$ были доказаны соответственно Волмэном [131] и Н.Б. Веденисовьм [130]. Ю.М. Смирнов [106] показал, что эти соотношения не переносятся на малую индуктивную размерность ind, ответив тем самым отрицательно на вопрос Веденисова. Контрпримером является пространство $S$ из замечания 2.6. В 1957 г. Ю.М. Смирнов [111] доказал, что для размерности ind вообще не выполняется теорема компактификации. А именно, он привел пример нормального пространства $X$ размерности ind $X=1$ такого, что ind $b X>1$ для любой компактификации $b X$. Этот результат в 1982 г. был усилен ван Миллом и Пшимусиньским [71], построившими совершенно нормальное пространство $X$ размерности ind $X=1$, которое обладает следуюшим свойством: если $Y \supset X$ - финально компактное пространство, то размерность ind $Y$ не определена.

2.9. Вопрос. Всякое ли метризуемое пространство $X$ имеет компактификацию $b X$ размерности ind $X=$ ind $b X$ ?

Известен лишь тривиальньй ответ на этот вопрос в случае ind $X=0$. Что касается размерностей $\operatorname{dim}$ и Ind, Е. Г. Скляренко [103] доказал существование компактификаций данного веса и данной размерности для dim, а Б. А. Пасьнков ([84], [5; приложение]) - для Ind. C. А. Богатый [10] показал, что для метрического пространства $X$ неравенство $\operatorname{dim} X \leqslant n$ равносильно сушествованию компактного расширения $b X$, нульмерно отображаюшегося в $n$-мерньй куб, в котором замыкания любых двух подмножеств $X$, находящихся на положительном расстоянии друг от друга, не пересекаются. Всякое такое расширение $b X$ представляется в виде объединения $n+1$ нульмерного подмножества, является замкнутым $(n+1)$-кратньм образом некоторого нульмерного компакта и для него $\operatorname{dim} b X=\operatorname{ind} b X=\operatorname{Ind} b X$.

Возвращаясь к стоун-чеховским компактификациям, отметим следующий результат Ю.М. Смирнова [106].

2.10. ПРЕДЛОЖЕНИЕ. Пусть $X$ - нормальное пространство, обладающее тем свойством, что $\operatorname{Ind}\left(F_{1} \cup F_{2}\right) \leqslant n$ для любых двух замкнутых подмножеств $F_{i}$ пространства $X$ размерности Ind $F_{i} \leqslant n$. Тогда

$$
\text { ind } \beta X=\text { Ind } X \text {. }
$$

Отсюда Ю. М. Смирнов вьвел справедливость равенства (2.7) для совершенно нормальных пространств $X$ и равенство

$$
\text { ind } X=\operatorname{ind} \beta X=\text { Ind } X
$$

для совершенно нормальных финально компактных пространств $X$. Энгелькинг [28] распространил равенство (2.7) на сильно наследственно нормальные пространства.

В 1958 г. Е.Г. Скляренко [102] доказал, что тихоновское пространство счетного типа (всякий компакт содержится в $G_{\delta}$-компакте) имеет компактификацию с нульмерным наростом тогда и только тогда, когда оно периферически компактно или, что 
то же самое, семибикомпактно. Тогда же Ю.М. Смирнов [112] построил пример не периферически компактного пространства, имеющего компактификацию с индуктивно-нульмерным наростом. В работах [119]-[122] Ю. М. Смирнов исследовал вопрос о размерности наростов компактификащий близостных и топологических пространств. Приведем здесь основной разультат, относяшийся к топологическим пространствам.

2.11. ТеОРемА. Тихоновское пространство $X$ обладает компактификацией $b X c \operatorname{dim}(b X \backslash X) \leqslant n$ тогда и только тогда, когда у него существует структура окаймлений кратности $\leqslant n+1$ с базисным свойством.

Размерность здесь определяется посредством конечных открытых покрытий, продолжаемых на стоун-чеховскую компактификацию. Окаймлением пространства $X$ называется такая конечная система $\left\{U_{1}, \ldots, U_{s}\right\}$ его открытых подмножеств, что

1) $X \backslash \bigcup U_{i}-$ компакт;

2) для любой окрестности $O\left(X \backslash \bigcup U_{i}\right)$ семейство $\left\{O\left(X \backslash \bigcup U_{i}\right), U_{1}, \ldots, U_{s}\right\}$ является покрытием, продолжаемьм на $\beta X$.

Семейство Г окаймлений пространства $X$ называется структурой, если для любых $\gamma_{1}, \gamma_{2} \in \Gamma$ сушествует $\gamma_{3} \in \Gamma$, звездно вписанное и в $\gamma_{1}$, и в $\gamma_{2}$. Наконец, структура Г обладает базисным свойством, если для любой точки $x \in X$ и любой ее окрестности $O x$ сушествуют окрестность $U x$ и окаймление $\gamma$ такие, что $S t_{\gamma} U x \subset O x$.

Ю.М. Смирнов заложил основы теории размерности пространств близости. Поскольку это направление выходит за рамки нашего обзора, упомянем здесь лишь основные по этой тематике работы самого Ю.М. Смирнова ([108], [109]) и его главного последователя Исбелла ([52]-[55]).

Более подробно остановимся на метрической размерности, которая также не является топологическим инвариантом. Это понятие было введено П. С. Александровым в начале 30-х годов прошлого века, но систематическое его исследование началось в 1956 г. в работе Ю.М. Смирнова [110]. Метрической размерностью $\mu \operatorname{dim} X$ метрического пространства $X$ назьвается наименьшее из чисел $n \geqslant 0$, обладаюших тем свойством, что в пространстве $X$ при любом $\varepsilon>0$ имеется открытое $\varepsilon$-покрытие кратности $n+1$.

Ю.М. Смирнов [110] показал, что метрическая размерность множества $X$, лежащего в евклидовом пространстве $\mathbb{R}^{m}$, может быть определена как наименшшее целое $n$, обладаюшее тем свойством, что множество $X$ может быть при любом $\varepsilon$ посредством $\varepsilon$-сдвига отображено в $n$-мерньй полиэдр (таково было определение Александрова). Поэтому известньй пример К.А. Ситникова ([100], [101]) двумерного множества $M \subset \mathbb{R}^{3}$, которое при любом $\varepsilon$ допускает $\varepsilon$-сдвиг в одномерный полиэдр, является примером двумерного множества метрической размерности 1. Для любого метрического пространства $X$ имеем

$$
\mu \operatorname{dim} X \leqslant \operatorname{dim} X .
$$

Это вытекает из знаменитой теоремы Даукера [19].

2.12. ТеоремА. Для нормального пространства $X$ следующие условия равносильнь:

(1) $\operatorname{dim} X \leqslant n$;

(2) всякое локально конечное открытое покрытие пространства $X$ можно ужать до открытого покрытия кратности $\leqslant n+1$; 
(3) всякое звездно конечное открытое покрытие пространства $X$ мохсн ужсать до открытого покрытия кратности $\leqslant n+1$.

Катетов [61] дополнил неравенство (2.9) до формулы

$$
\mu \operatorname{dim} X \leqslant \operatorname{dim} X \leqslant 2 \mu \operatorname{dim} X
$$

неулучшаемой в силу упомянутого вьше примера Ситникова. Интерес представляет и следующая теорема Ситникова [101]: если $X \subset \mathbb{R}^{m}$ и $\operatorname{dim} X=\operatorname{dim} \mathrm{Cl}(X)$, то $\mu \operatorname{dim} X=\operatorname{dim} X$.

Ю.М. Смирнов и его ученик В.И. Егоров ([23]-[25], [110]) построили основы метрической теории размерности. Были доказаны, в частности, аналоги таких фундаментальных утверждений, как аддиционная теорема, теорема о перегородках и теорема о сушественных отображениях. Эти и более обшие результаты о размерности метрических и равномерных пространств были освящены также в обзорном докладе [124].

После знаменитой теоремы Куратовского-Дугунджи Ю.М. Смирнов дал новый импульс для исследований на стыке теории размерности и теории экстензоров. Так, решая проблему П. С. Александрова об уплотнениях на компакты связного, локально связного, полного метрического $\sigma$-компактного пространства, Ю.М. Смирнов [123] получил теорему о продолжении отображения в окружность с любого слагаемого компактного счетного объединения компактов, имеющих нульмерные попарные пересечения. Впоследствии Н. Хаджииванов в ответ на вопрос Ю. М. Смирнова получил ([45], [46]) многомерный вариант этой теоремы.

2.13. ТеоремА. Если компакт $X$ является счетным облединением компактов, попарные пересечения которых самое большее $(n-1)$-мерны, то всякое отображение каждого из них в п-мерную сферу продолжаемо на все $X$.

Б. Т. Левшенко и Ю. М. Смирнов доказали [70], что всякое замкнутое подмножество нульмерного метрического пространства является ретрактом всего пространства. Энгелькинг показал [27], что сушествует даже замкнутая ретракция. В общем случае Б. Т. Левшенко [69] доказал, что для всякого замкнутого подмножества $A$ метрического пространства $X$ со свойством $\operatorname{dim}(X \backslash A) \leqslant n$ существует такое множество $F \subset X \backslash A$, замкнутое в $X \backslash A$ и типа $F_{\sigma}$ в $X$, что $\operatorname{dim}(X \backslash F) \leqslant n-1$ и $X \backslash F$ ретрагируется на $A$. А. Б. Куровский и Ю. М. Смирнов показали [63], что для метрических пространств утверждение Б. Т. Левшенко может быть взято в качестве индуктивного определения размерностно-ретракционного инварианта Ird и доказали равенство $\operatorname{Ird} X=\operatorname{dim} X$. С. А. Богатый показал [9], что теорема Энгелькинга переносится и на ситуащию второй теоремы Левшенко, т.е. существует замкнутая ретракция множества $F$ с указанными выше свойствами. В [9] было предложено утверждение, включающее в себя теорему Левшенко, теоремы Куратовского-Дугунджи, характеризуюшие $\operatorname{AE}(n)$ - и $\operatorname{ANE}(n)$-пространства, и теорему Эйленберга-Борсука. В.А. Никифиров получил [80] селекционный вариант теоремы Эйленберга-Борсука, включающий в себя конечномерную селекционную теорему Майкла. С. М. Агеев и С. А. Богатьй построили [1] “универсальные” препятствия. Г. М. Непомнящий и Ю. М. Смирнов [79] изучали ретракты в категории отображений. 


\section{§ 3. Бесконечномерные пространства}

Нормальное пространство $X$ назьвается счетномерным, если оно может быть представлено в виде счетного объединения конечномерных подпространств. Нормальное пространство, не являюшееся счетномерным, назьвается несчетномерныл. Еще П. С. Урысон высказал предположение [129; гл. 6], что гильбертов куб не может быть представлен в виде счетной суммы своих нульмерных подпространств. Эта гипотеза нашла свое подтверждение в работе Гуревича [49], где тот дал определение счетномерного пространства (в классе сепарабельных метрических пространств) и доказал несчетномерность гильбертова куба.

Из аддиционной теоремы для размерности $\operatorname{dim}($ неравенство (2.3)) вытекает

3.1. ПРЕДЛОЖЕНИЕ. Наследственно нормальное пространство $X$ счетномерно тогда и только тогда, когда $X$ может быть представлено в виде суммы растущей последовательности подпространств $X_{n}$ размерности $\operatorname{dim} X_{n} \leqslant n$, $n=1,2, \ldots$.

Из теоремы Катетова (теорема 2.4) вытекает

3.2. ТеОРема. Метризуемое пространство $X$ счетномерно тогда и только тогда, когда $X$ может быть представлено в виде суммы последовательности подпространств $X_{n}$ размерности $\operatorname{dim} X_{n} \leqslant 0, n=1,2, \ldots$.

Нагами [75] усилил теорему 3.2. Он доказал, что последовательность подпространств $X_{i}$ можно считать растущей.

3.3. ЗАмЕчАнИЕ. Условие метризуемости пространства $X$ в теореме 3.2 существенно. В самом деле, в 1986 г. А. А. Одинщов [82] в предположении континуум-гипотезы построил одномерньй (и даже змеевидньй) совершенно нормальньй компакт, которьй не представляется в виде счетной суммы нульмерных подпространств. Он же заметил, что, ослабляя совершенную нормальность до 1-й аксиомы счетности, можно построить такой компакт и в ZFC. Более общий подход к построению пространств такого рода (правда, размерности $\geqslant 2$ ) связан с компактами без промежмтолиьы размерностей, т.е. такими конечномерными компактами $X$ размерности $\operatorname{dim} X \geqslant 2$, что для любых непустых замкнутых множеств $F \subset X$ имеем либо $\operatorname{dim} F=0$, либо $\operatorname{dim} F=\operatorname{dim} X$. В 1973 г. автор [29] для всякого $n \geqslant 2$ построил такой $n$-мерный компакт $B_{n}$ с 1-й аксиомой счетности. Затем были построены совершенно нормальные компакты без промежуточных размерностей. Сначала это сделал автор ([30], [32]) в предположении принципа Йенсена $\diamond$. Потом Н. В. Савинов [98] построил такой компакт в СН. Наконец, в 1978 г. автор [33] предложил более простой и общий способ построения в СН совершенно нормальных компактов без промежуточных размерностей.

Но какое же отношение к теореме 3.2 имеют эти компакты? Ответ на этот вопрос дает следуюшее утверждение.

3.4. ПРЕДЛОЖЕНИЕ [34]. Если наследственно нормальный компакт $X$ размерности $\operatorname{dim} X>0$ является счетной суммой нульмерных подпространств, то $X$ содержит замкнутое одномерное множество. 
3.5. СлЕДСТВИЕ. Никакой наследственно нормальный компакт без промежуточных размерностей не является счетной суммой нульмерных подпространств.

Последний результат можно усилить.

3.6. ПРЕДЛОЖЕНИЕ. Никакой компакт без промежуточных размерностей не является счетной суммой индуктивно нульмерных подпространств.

ДокАЗАТЕльство. Пусть $X-n$-мерньй компакт без промежуточных размерностей. По теореме 1.4 можно считать, что $X$ является $n$-мерным канторовым многообразием. Предположим, что $X$ является суммой индуктивно нульмерных подпространств $X_{i}, i \in \omega$. Возьмем произвольно точку $x_{0} \in X_{0}$ и отличную от нее точку $x \in X$. В силу индуктивной нульмерности пространства $X_{0}$ в окрестности $X \backslash\left\{x_{0}\right\}$ точки $x_{0}$ существует такая окрестность $O x_{0}$, что $X_{0} \cap \operatorname{Bd} O x_{0}=\varnothing$. Значит, $\operatorname{Bd} O x_{0} \subset \bigcup\left\{X_{i}: i \geqslant 1\right\}$.

Поскольку $X$ является $n$-мерным канторовьм многообразием, $\operatorname{dim} \operatorname{Bd} O x_{0} \geqslant$ $n-1 \geqslant 1$. Следовательно, $\operatorname{dim} \mathrm{Bd} O x_{0}=n$. По той же теореме 1.4 сушествует $n$-мерное канторово многообразие $C_{0} \subset \operatorname{Bd} O x_{0}$. По выбору окрестности $O x_{0}$ имеем $C_{0} \cap X_{0}=\varnothing$. Продолжая этот процесс, получаем вложенную последовательность таких $n$-мерных канторовых многообразий $C_{i}$, что $C_{i} \cap X_{i}=\varnothing$. Тогда $\bigcap C_{i}$ не пусто и $\left(\bigcap C_{i}\right) \cap\left(\bigcup X_{i}\right)=\varnothing$. Полученное противоречие и доказьвает предложение 3.6.

В связи с вышесказанньм возникают следующие вопросы.

3.7. Вопрос. Совместимо ли с ZFC, что всякий одномерный совершенно (наследственно) нормальньй компакт есть сумма счетного числа нульмерных подпространств?

3.8. Вопрос. Сушествует ли в ZFC пример совершенно (наследственно) нормального компакта без промежуточных размерностей?

3.9. Вопрос. Сушествует ли в ZFC совершенно (наследственно) нормальное счетномерное (компактное) пространство, не являюшееся суммой счетного числа нульмерных множеств?

По поводу теоремы 3.2 отметим также следующий результат Ю. М. Смирнова.

3.10. ТЕорема. Любое метрическое пространство $X$ мохет быть представлено в виде облединения растущей трансфинитной последовательности подпространств $X_{\alpha}, \alpha \in \omega_{1}$, размерности $\operatorname{dim} X_{\alpha} \leqslant 0$.

Это утверждение для сепарабельных пространств доказано в [113]. По поводу общего случая см. [28; Problem 5.1.В.(a)].

Нормальное пространство назьвается слабо счетномерным, если оно может быть представлено в виде суммы счетного числа замкнутых конечномерных подпространств.

3.11. ЗАмЕчАНИЕ. В англоязычной литературе такие пространства назьваются сильно счетномерными. Это, по-видимому, связано с тем, что при абстрактном определении класса пространств со свойством $\mathscr{P}$ естественно считать, что всякое пространство с сильньм свойством $\mathscr{P}$ должно быть пространством со свойством $\mathscr{P}$. Например, всякое сильно паракомпактное пространство паракомпактно. Но когда речь 
идет о бесконечномерных пространствах, такой подход не является абсолютно верным. В отечественной школе обшей топологии эти пространства назьваются слабо счетномерными, потому что они "менее" бесконечномерны, чем счетномерные пространства. То же происходит и в случае слабой и сильной бесконечномерности (см. ниже).

Ясно, что всякое слабо счетномерное пространство счетномерно. Из теоремы суммы для лебеговой размерности вытекает

3.12. ПРЕДЛОЖЕНИЕ. Нормальное пространство слабо счетномерно тогда и только тогда, когда оно может быть представлено в виде суммы счетной растущей последовательности конечномерных замкнутых подпространств.

В 1928 г. Гуревич [49] объявил о существовании счетномерного метризуемого компакта, которьй не представляется в виде счетной суммы конечномерных подкомпактов. Но первьй пример такого компакта был опубликован в 1959 г. Ю. М. Смирновым [115]. В этой статье, а также в появившейся годом позже статье Нагаты [76] было начато систематическое исследование слабо счетномерных пространств. В частности, там было показано, что подмножество $K_{\omega}$ гильбертова куба $I^{\omega_{0}}$, состоящее из всех точек, лишш конечное число координат которых отлично от нуля, является универсальным пространством для класса всех слабо счетномерных сепарабельных метризуемых пространств.

Е. Г. Скляренко [105] доказал, что пространство $K_{\omega}$ не имеет счетномерных метризуемых компактификаций, в то время как по теореме Гуревича [49] всякое полное счетномерное сепарабельное метрическое пространство имеет счетномерную метризуемую компактификацию. Более того, всякое полное слабо счетномерное сепарабельное метрическое пространство имеет слабо счетномерную метризуемую компактификацию [99]. Напомним также одну теорему А. В. Зарелуа [136]: если стоун-чеховская компактификация $\beta X$ нормального пространства $X$ слабо счетномерна, то пространство $X$ имеет слабо счетномерную компактификацию $b X$ веса $w(X)$. Как показал Е.Г. Скляренко [104], пространство $K_{\omega}$ не имеет никаких слабо счетномерных компактификаций. Более 30 лет остается нерешенным

3.13. Вопрос. Сушествует ли у $K_{\omega}$ счетномерная компактификация?

В связи с этим вопросом стоит отметить, что стоун-чеховская компактификация $\beta K_{\omega}$ несчетномерна (это вытекает из результатов ван Дауэна [18] и Э. Поль [89]).

Возвращаясь к универсальным пространствам, напомним, что Нагата доказал существование универсальных пространств для следуюших классов пространств: счетномерные сепарабельные метризуемые пространства [76], счетномерные метризуемые пространства веса $\leqslant \tau[78]$, счетномерные сильно метризуемые пространства веса $\leqslant \tau$ [76], слабо счетномерные нормальные пространства веса $\leqslant \tau$ [77], слабо счетномерные сильно метризуемые пространства веса $\leqslant \tau$ [76]. А.В. Архангельский [7] построил универсальное пространство для класса всех слабо счетномерных метризуемых пространств веса $\leqslant \tau$. Б. А. Пасьнков [84] доказал, что в классе слабо счетномерных нормальных пространств веса $\leqslant \tau$ существует универсальное пространство, являюшееся суммой счетного числа конечномерных компактов. В то же время нерешенными остаются следуюшие вопросы. 
3.14. Вопрос. Существует ли универсальное пространство для класса всех счетномерных нормальных пространств веса $\leqslant \tau$ для несчетного $\tau$ ?

3.15. Вопрос. Существует ли счетномерное нормальное пространство несчетного веса $\tau$, содержашее все счетномерные компакты веса $\leqslant \tau$ ?

Характеризация размерности $\operatorname{dim}$ посредством перегородок (теорема 1.1) легла в основу следуюшего определения. Нормальное пространство $X$ назьвается слабо бесконечномерным м, если для любой последовательности $\left(A_{i}, B_{i}\right), i \in \omega$, пар дизъюнктных замкнутых его подмножеств найдутся такие перегородки $C_{i}$ в $X$ между $A_{i}$ и $B_{i}$, что $\bigcap\left\{C_{i}: i \in \omega\right\}=\varnothing$. Нормальное пространство, не являюшееся слабо бесконечномерным, называется сильно бесконечномерным. Это определение было впервые рассмотрено в 1948 г. П. С. Александровым [4].

Через десять лет Ю.М. Смирнов дал другой вариант определения бесконечномерности. Нормальное пространство $X$ называется $S$-слабо бесконечномерным, если для любой последовательности $\left(A_{i}, B_{i}\right), i \in \omega$, пар дизъюнктных замкнутых его подмножеств найдутся такие перегородки $C_{i}$ в $X$ между $A_{i}$ и $B_{i}$, что некоторое конечное подсемейство перегородок $C_{i}$ имеет пустое пересечение. Нормальное пространство, не являюшееся $S$-слабо бесконечномерным, назьвается $S$-сильно бесконечномерным. После этого слабо (сильно) бесконечномерные пространства стали иногда называться $A$-слабо ( $A$-сильно) бесконечномерными. Ясно, что всякое $S$-слабо бесконечномерное пространство слабо бесконечномерно и что для компактов (даже для счетнокомпактных пространств) эти понятия совпадают.

Детальное исследование свойств слабой и сильной бесконечномерности было начато в работах Б.Т. Левшенко [66], Е.Г. Скляренко ([104], [105]) и Ю.М. Смирнова [114]. Интересный комментарий к определению $S$-слабой бесконечномерности дал Е.Г. Скляренко. Он показал [104], что для любого метризуемого компакта $X$ и для любого несчетного семейства $P$ пар дизъюнктных замкнутых подмножеств этого компакта сушествуют две пары $\left(A_{1}, B_{1}\right),\left(A_{2}, B_{2}\right)$ из семейства $P$ и непересекаюшиеся перегородки $C_{1}$ и $C_{2}$ между $A_{1}$ и $B_{1}$ и между $A_{2}$ и $B_{2}$ соответственно.

Были доказаны [66] теоремы счетной суммы для слабо бесконечномерных пространств (произвольные слагаемые в наследственно нормальных пространствах и замкнутые слагаемые в нормальных и счетно паракомпактных пространствах). Из этих теорем вытекает, что слабо бесконечномерным является всякое наследственно нормальное счетномерное пространство и всякое нормальное счетно паракомпактное слабо бесконечномерное пространство. На основе этих теорем можно привести большое количество простых примеров слабо бесконечномерных пространств, являюшихся $S$-сильно бесконечномерными. Одним из таких пространств будет дискретная сумма кубов $I^{n}, n \in \omega$. Ю.М. Смирнов показал [117], что всякое бесконечномерное метрическое пространство $X$ содержит $S$-сильно бесконечномерное пространство $Y$. Более того, такое $Y$ можно получить выбрасьванием из $X$ не более одной точки. Слабая бесконечномерность и $S$-слабая бесконечномерность различаются и на уровне многообразий. Так, В. А. Дулев [22] в предположении СН построил совершенно нормальное наследственно сепарабельное дифференцируемое 4-многообразие, которое слабо бесконечномерно, но $S$-сильно бесконечномерно.

Е.Г. Скляренко [105] показал, что конечная сумма замкнутых $S$-слабо бесконечномерных слагаемых будет $S$-слабо бесконечномерна. Б.Т. Левшенко [66] 
охарактеризовал $S$-сильно бесконечномерные пространства посредством наличия у них существенных отображений на гильбертов куб $Q \equiv I^{\omega_{0}}$. При этом отображение $f: X \rightarrow I^{\omega_{0}}$ называется существенным, если сушественно всякое отображение $p_{n} \circ f$, где $p_{n}: I^{\omega_{0}} \rightarrow I^{n}-$ проектирование на грань. Е.Г. Скляренко [104] доказал, что упомянутое вьше пространство $K_{\omega}$ не имеет слабо бесконечномерных компактификаций. Это является еще одним комментарием к вопросу 3.13. Е. Г. Скляренко описал строение $S$-слабо бесконечномерных пространств [105]. В частности, имеет место

3.16. ТеоремА. Слабо паракомпактное нормальное пространство $X$ является S-слабо бесконечномерным тогда и только тогда, когда существует такой компакт $K \subset X$, что всякое замкнутое в $X$ множество $F \subset X \backslash K$ конечномерно.

Определение малой трансфинитной размерности ind повторяет определение малой индуктивной размерности с заменой неотрицательных целых чисел порядковыми числами. Возможность распространения малой индуктивной размерности на трансфинитные числа была отмечена в 1922 г. П. С. Урысоном [128]. В первой главе своего "Мемуара о канторовых многообразиях" он писал:

"Вместо бесконечной размерности можно было бы ввести трансфинитные размерности для всех чисел второго класса и, может быть, даже дальше, потому что размерность (согласно ее определению) есть порядковое число. Впрочем, это распространение, по крайней мере в данньй момент, кажется мне лишенным интереса, тем более, что даже в этом случае останутся, по-видимому, множества, не укладывающиеся в классификацию (области гильбертова пространства)".

Формальное определение малой трансфинитной размерности было дано Гуревичем в работе [49], где получены первые результаты об этом инварианте. Было доказано, в частности, что:

1) ind $X<\omega_{1}$ для всякого сепарабельного метрического пространства $X$;

2) всякое сепарабельное метрическое пространство, имеющее малую трансфинитную размерность, счетномерно;

3) всякое счетномерное полное метрическое пространство имеет малую трансфинитную размерность.

Последнее утверждение было доказано для компактных пространств. Доказательство для полных метрических пространств появилось в книге [51]. Из утверждений 2) и 3) вытекает

3.17. Теорема. Сепарабельное полное метрическое пространство имеет малую трансфинитную размерность тогда и только тогда, когда оно счетномерHO.

Полнота пространства в утверждении 3) и в теореме 3.17 сушественна. Это вытекает из упомянутой выше теоремы Е.Г. Скляренко [104] о том, что счетномерное пространство $K_{\omega}$ не имеет счетномерных метризуемых компактификащий, и из следующей теоремы [28; 7.2.21], анонсированной еше Гуревичем [49].

3.18. ТеОремА. Сепарабельное метрическое пространство имеет малую трансфинитную размерность тогда и только тогда, когда оно имеет счетномерную метризуемую компактификацию. 
В связи с теоремой Гуревича о счетномерности всякого сепарабельного метрического пространства, имеюшего малую трансфинитную размерность (утверждение 2)), отметим, что Ю.М. Смирнов ([116]-[118]) распространил ее на сильно метризуемые пространства, а Э. Поль [90] построила совершенно нормальное несчетномерное пространство, имеюшее малую трансфинитную размерность. Предполагая континуум-гипотезу, автор [38] построил совершенно нормальное наследственно сепарабельное сильно бесконечномерное дифференцируемое 4-многообразие $M$. Пространство $M$, будучи 4-многообразием, имеет малую индуктивную (трансфинитную) размерность, равную 4. Долгое время остаются нерешенными следуюшие вопросы.

3.19. Вопрос. Будет ли счетномерным всякое метрическое пространство, малая трансфинитная размерность которого определена?

3.20. Вопрос. Будет ли счетномерным всякий (совершенно нормальный) компакт, малая трансфиннитная размерность которого определена?

По поводу последнего вопроса можно упомянуть теорему Б. Т. Левшенко [67] о том, что всякое сильно паракомпактное пространство, которое имеет малую трансфинитную размерность, слабо бесконечномерно.

Большая трансфинитная размерность Ind была определена в 1959 г. Ю. М. Смирновым в работе [115]. Сформулируем основные утверждения, содержашиеся в этой работе.

3.21. ТеОремА. Если компакт $X$ веса $w(X) \leqslant \omega_{\alpha}$ имеет большую трансфинитную размерность, то Ind $X<\omega_{\alpha+1}$.

3.22. СлЕДСТВИЕ. Если метризуемый компакт $X$ имеет большую трансфинитную размерность, то Ind $X<\omega_{1}$.

3.23. ТЕОРемА. Для любого нормального пространства $X$ имеем ind $X \leqslant$ Ind $X$

3.24. ПРЕДЛОЖЕНИЕ. Если нормальное пространство $X$ является дискретной суммой подпространств $X_{n}$ размерности Ind $X_{n} \geqslant n, n \in \omega$, то большая трансфинитная размерность пространства $X$ не определена.

3.25. ТеОрема. Всякое счетномерное компактное метризуемое пространство имеет больиую трансфинитную размерность.

В этой работе были также построены получившие большую известность компакmbl Смирнова: такие метризуемые слабо счетномерные компакты $S_{\alpha}, \alpha<\omega_{1}$, что

$$
\text { Ind } S_{\alpha}=\alpha \text {. }
$$

Из теорем $3.17,3.23$ и 3.25 вытекает

3.26. ТЕОРемА. Для метризуемого компакта $X$ следующие условия равносильны:

1) определена малая трансфинитная размерность ind $X$;

2) определена большая трансфинитная размерность Ind $X$;

3) компакт $X$ счетномерен.

Из самого существования компактов Смирнова вытекает 
3.27. ТЕОРемА. В классе сепарабельных метризуемых пространств, для которых определена большая трансфинитная размерность, не существует универсального пространства.

Если же добавить еще теорему 3.25 , то получим следующие два утверждения.

3.28. ТЕОремА. Не существует универсального пространства в классе счетномерных метризуемых компактов.

3.29. ТЕОремА. Не существует универсального пространства в классе слабо счетномерных метризуемых компактов.

Последние две теоремы усилил Р. Поль [93], который доказал, что всякое полное сепарабельное метрическое пространство, топологически содержашее все компакты Смирнова $S_{\alpha}$, содержит и гильбертов куб. Компакты Смирнова позволили построить 4-многообразия произвольной счетной большой трансфинитной размерности. Более точно, в [38] было доказано следующее утверждение.

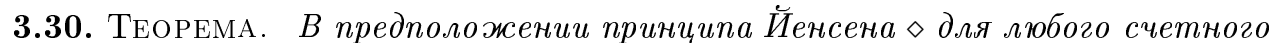
трансфинитного числа $\alpha \geqslant 5$ существует совершенно нормальное наследственно сепарабельное дифференцируемое 4-многообразие $M_{\alpha}$ размерности Ind $M_{\alpha}=\alpha$.

Напомним также следующие результаты Ю. М. Смирнова из работ [117] и [118] .

3.31. ТЕОРемА. Если метризуемое пространство $X$ имеет большую трансфинитную размерность, то Ind $X<\omega_{1}$.

3.32. ТЕОремА. Если метризуемое пространство $X$ имеет большую трансфинитную размерность, то $X$ счетномерно.

3.33. ТЕОремА. Если наследственно нормальное пространство имеет большую трансфинитную размерность, то оно слабо бесконечномерно.

А. Ч. Чигогидзе [16] освободился от условия наследственной нормальности в последнем утверждении. Из предложения 3.24 вытекает, что классы метризуемых пространств (и даже сепарабельных полно метризуемых), для которых определены соответственно малая и большая трансфинитные размерности, существенно различаются. В то же время, если для какого-нибудь компакта определена малая трансфинитная размерность, то для него определена и большая трансфинитная размерность. В наследственно нормальном случае это показал Б. Т. Левшенко [68], в общем случае автор [34]. Но, как показывают компакты без промежуточных размерностей (замечание 3.3$)$, в полной мере распространить теорему 3.26 на неметризуемые компакты нельзя.

\section{$\S$ 4. Многообразия $M^{4, C}$}

Спектр $S^{\varphi}$. В 1952 г. Андерсон [6] построил монотонное открытое отображение $f: M \rightarrow Q$ одномерного континуума $M$ на гильбертов куб. Для нас будет несушественно, что отображение $f$ открыто. Важно будет то, что все его слои $f^{-1}(q), q \in Q$, являются невырожденными континуумами. 
Вложим континуум $M$ в сферу $S^{3}$, ограничивающую замкнутый шар $B^{4}$. Возьмем произвольный компакт $C$, лежаший в $Q$, и определим разбиение $\mathscr{D}_{C}$ шара $B^{4}$ следуюшим образом:

$$
\mathscr{D}_{C}=\left\{f^{-1}(q): q \in C\right\} \cup\left\{\{b\}: b \in B^{4} \backslash f^{-1}(C)\right\} .
$$

Ясно, что это разбиение полунепрерьвно сверху. Факторпространство $B^{4} / \mathscr{D}_{C}$ обозначим через $B_{C}^{4}$, факторное отображение $B^{4} \rightarrow B_{C}^{4}$ обозначим через $\varphi \equiv \varphi_{C}$. Положим $S_{C}^{3}=\varphi\left(S^{3}\right)$. Заметим, что компакт $C$ естественно гомеоморфен компакту $\varphi\left(f^{-1}(C)\right)$ и, следовательно, вложен в компакт $S_{C}^{3}$.

Точку $y \in S_{C}^{3}$ назовем точкой первого рода, если множество $\varphi^{-1}(y)$ одноточечно, и точкой второго рода, если $\varphi^{-1}(y)$ совпадает с одним из континуумов $f^{-1}(q)$, $q \in Q$. Пусть $\left\{s_{\gamma}: \gamma<\omega_{1}\right\}$ - какая-нибудь нумерация точек множества $S_{C}^{3}$, сушествуюшая согласно континуум-гипотезе.

Теперь, практически дословно повторяя рассуждения из [37], построим непрерьвньй обратньй спектр $S^{\varphi}=\left\{X_{\beta}, \pi_{\gamma}^{\beta}: \gamma \leqslant \beta<\omega_{1}\right\}$. Но для этого надо напомнить старые обозначения некоторых множеств и ввести новые. Пусть $S_{C}^{3}, Z_{0}, J_{\alpha}$ - попарно непересекаюшиеся множества мошности континуум, где $1 \leqslant \alpha<\omega_{1}, \alpha$ - изолированньй трансфинит. Для счетного трансфинита $\beta$ полагаем

$$
\begin{gathered}
Z_{\beta}=Z_{0} \cup\left\{\bigcup\left\{J_{\alpha}: \alpha \leqslant \beta\right\}\right\}, \\
X_{\beta}=Z_{\beta} \cup S_{C}^{3} .
\end{gathered}
$$

Заметим, что при $\gamma<\beta$ мы имеем

$$
X_{\beta}=X_{\gamma} \cup\left\{\bigcup\left\{J_{\alpha}: \gamma+1 \leqslant \alpha \leqslant \beta\right\}\right\} .
$$

Полагаем $Z=\bigcup\left\{Z_{\alpha}: \alpha<\omega_{1}\right\}$ и, применяя континуум-гипотезу, берем такую нумерацию $\left\{A_{\delta}: \delta<\omega_{1}\right\}$ всех счетных подмножеств множества $Z$, что

$$
A_{\delta} \subset Z_{\gamma} \text { при } \delta \leqslant \gamma
$$

Определим теперь отображения $\pi_{\gamma}^{\beta}: X_{\beta} \rightarrow X_{\gamma}$ так, чтобы система

$$
S^{\varphi}=\left\{X_{\beta}, \pi_{\gamma}^{\beta}: \gamma \leqslant \beta<\omega_{1}\right\}
$$

была непрерьвньм обратным спектром множеств. Эти отображения определяются следуюшим условием.

1. $\pi_{\gamma}^{\beta}$ тождественно на $X_{\gamma}$ и переводит множество $J_{\alpha}$ в точку $s_{\alpha-1}$ при $\gamma+1 \leqslant \alpha \leqslant \beta$ (здесь мы используем изолированность $\alpha$ ).

Непрерьвность спектра $S^{\varphi}$ как спектра множеств вытекает из равенства (4.1). Теперь, как и в [37], топологизируем спектр $S^{\varphi}$, одновременно определяя топологию $\mathscr{T}_{\beta}$ на $X_{\beta}$ и дифференциальную структуру $\mathscr{D}_{\beta}$ на $Z_{\beta}$ так, что:

2. Спектр $S^{\varphi}$ топологически непрерывен.

3. $X_{\beta}$ гомеоморфно $B_{C}^{4}$ для любого $\beta<\omega_{1}$.

Отметим, что спектр $S^{\varphi}$ топологически непрерьвен, поскольку он непрерьвен как спектр множеств и состоит из компактов согласно условию 3. 
4. $X_{\beta} \backslash Z_{\beta}$ гомеоморфно $S_{C}^{3}$.

5. $\pi_{\gamma}^{\beta}$ есть почти гомеоморфизм при всех $\gamma<\beta$.

Свойство 5 для соседних отображений будет вьполняться по построению, для их конечных композиций - согласно предложению 1.12 , а для предельных $\beta$-в силу свойства 2 и леммы 1.11.

6. Если $\gamma<\beta$, то $s_{\gamma}$ является единственной точкой неоднозначности отображения $\pi_{\gamma}^{\gamma+1}$ и

$$
\left(\pi_{\gamma}^{\gamma+1}\right)^{-1}\left(s_{\gamma}\right)=\left\{s_{\gamma}\right\} \cup J_{\gamma+1} \equiv K_{\gamma+1}
$$

гомеоморфно отрезку $I$, когда $s_{\gamma}$ есть точка первого рода, и конусу над континуумом $f^{-1}\left(s_{\gamma}\right)=\varphi^{-1}\left(s_{\gamma}\right)$, когда $s_{\gamma}$ есть точка второго рода.

7. Если $\delta \leqslant \gamma<\beta$ и $s_{\gamma}$ является предельной точкой множества $A_{\delta}$ в $X_{\gamma}$, то всякая точка $x \in K_{\gamma+1}$ является предельной точкой множества $A_{\delta}$ в пространстве $\left(X_{\beta}, \mathscr{T}_{\beta}\right)$ (при этом следует иметь в виду, что $A_{\delta} \subset Z_{\gamma} \subset Z_{\beta}$ согласно условию (4.2)).

8. $\pi_{\gamma}^{\gamma+1} \mid\left(\pi_{\gamma}^{\gamma+1}\right)^{-1}\left(Z_{\gamma}\right)$ - гомеоморфизм.

9. Отображение $\pi_{\gamma}^{\beta} \mid S_{C}^{3}$ тождественно.

10. Многообразие $\left(Z_{\beta}, \mathscr{D}_{\beta}\right)$ диффеоморфно $\mathbb{R}^{4}$, т.е. атлас $\mathscr{D}_{\beta}$ содержит такую карту $\left(Z_{\beta}, \psi_{\beta}\right)$, что $\psi_{\beta}\left(Z_{\beta}\right)=\mathbb{R}^{4}$.

11. Если $\gamma<\beta$, то $\left(Z_{\gamma}, \psi_{\gamma}\right) \in \mathscr{D}_{\beta}$.

Чтобы читатель не был полностью зависим от работы [37], напомним вкратце построение спектра $S^{\varphi}$. Для вьполнения свойства 3 полагаем $X_{0}=B_{C}^{4}$. Открытый шар $B^{4} \backslash S^{3}$ обозначим через $O^{4}$. Ясно, что $O^{4}=B_{C}^{4} \backslash S_{C}^{3}$. Полагаем $Z_{0}=O^{4}$. В качестве $\mathscr{D}_{0}$ берем обычную гладкую структуру на $Z_{0}=O^{4}$, порожденную атласом, который состоит из единственной карты $\left(Z_{0}, \psi_{0}\right)$, где $\psi_{0}: O^{4} \rightarrow \mathbb{R}^{4}$ - какой-нибудь фиксированный диффеоморфизм.

Далее построение идет методом трансфинитной рекурсии. Если $\beta$ - предельньй трансфинит, то полагаем $X_{\beta}$ равным пределу обратного спектра $S_{\beta}^{\varphi}=\left\{X_{\gamma}, \pi_{\gamma^{\prime}}^{\gamma}, \beta\right\}$. В качестве отображения $\pi_{\gamma}^{\beta}$ берем сквозную проекцию $X_{\beta} \rightarrow X_{\gamma}$ спектра $S_{\gamma}^{\varphi}$. Свойства 2, 6-8 вьполнены автоматически. Вьполнение свойства 5 , влекушего свойство 3 , прокомментировано сразу после его формулировки. Свойства 4 и 9 вытекают из определения множеств $X_{\beta}, Z_{\beta}$ и свойства 1 . В качестве $\mathscr{D}_{\beta}$ берем дифференциальную структуру, порожденную атласом $\left\{\left(Z_{\gamma}, \psi_{\gamma}\right): \gamma<\beta\right\}$. Тогда свойство 11 вьполняется автоматически, а свойство 10 вытекает из теоремы 1.13 .

В случае $\beta=(\beta-1)+1$ применяем лемму 1.15 для $\mathscr{C}=\left\{A_{\delta}: \delta \leqslant \beta-1\right\}$, $y=s_{\beta-1}, B_{\varphi}^{n}=B_{C}^{4}, S_{\varphi}^{n-1}=S_{C}^{3}$ и $K$, равного отрезку или конусу над континуумом $\varphi^{-1}\left(s_{\beta-1}\right)$ в зависимости от рода точки $s_{\beta-1}$. В качестве $\pi_{\beta-1}^{\beta}$ берем отображение $g$ из леммы 1.15. Топологические свойства $2-9$ либо выполняются автоматически, либо вытекают из леммы 1.15 . В качестве $\mathscr{D}_{\beta}$ берем дифференциальную структуру на $Z_{\beta}$, порожденную атласом $\mathscr{D}_{\beta-1} \cup\left\{\left(Z_{\beta}, \psi_{\beta}\right)\right\}$, где отображение $\psi_{\beta}: O_{4} \rightarrow \mathbb{R}^{4}$ совпадает с первоначальньм диффеоморфизмом $\psi_{0}$. Семейство $\mathscr{D}_{\beta-1} \cup\left\{\left(Z_{\beta}, \psi_{\beta}\right)\right\}$ является атласом, так как отображение

$$
\pi_{\beta-1}^{\beta}: Z_{\beta} \backslash J_{\beta} \rightarrow Z_{\beta-1}
$$

есть дифффеоморфизм по свойству 6) леммы 1.15. 
Пространство $X^{\varphi}$ и многообразие $M^{4, C}$. Обозначим через $X=X^{\varphi}$ предел топологизированного обратного спектра $S^{\varphi}$ и положим $M^{4, C}=(Z, \mathscr{D})$, где $\mathscr{D}=$ $\bigcup\left\{\mathscr{D}_{\beta}: \beta<\omega_{1}\right\}$. Таким образом, $X$ - компакт, $M^{4, C}$ - дифференцируемое 4-многообразие, являюшееся открытым подмножеством $X$, а разность $X \backslash M^{4, C}$ гомеоморфна компакту $S_{C}^{3}$ в силу свойств 4 и 9 спектра $S^{\varphi}$.

Для $\beta<\omega_{1}$ пусть $\pi_{\beta}: X \rightarrow X_{\beta}=B_{C}^{4}-$ сквозная проекция спектра $S^{\varphi}$. Из свойства 6 спектра $S^{\varphi}$ вытекает, что:

(4.3) Если $x \in X_{0} \backslash S_{C}^{3}$, то $\pi_{0}^{-1}(x)$ состоит из одной точки.

(4.4) Если $s \in S_{C}^{3}$ есть точка первого рода, то множество $\pi_{0}^{-1}(s)$ гомеоморфно отрезку $I$.

(4.5) Если $s \in S_{C}^{3}$ есть точка второго рода, то множество $\pi_{0}^{-1}(s)$ гомеоморфио конусу над невырожденньм подконтинуумом континуума $M$.

Из (4.3)-(4.5) и предложения 1.7 получаем

(4.6) Компакт $X$ удовлетворяет первой аксиоме счетности.

Из свойства 7 спектра $S^{\varphi}$ вытекает

(4.7) Если $s_{\gamma}$ есть предельная точка множества $A_{\delta}$ в $X_{\gamma}$ при $\delta \leqslant \gamma$, то $\pi_{\gamma}^{-1}\left(s_{\gamma}\right) \subset$ $\mathrm{Cl}_{X}\left(A_{\delta}\right)$.

Из определения множества $Z$ и свойства 10 спектра $S^{\varphi}$ получаем

(4.8) Многообразие $M^{4, C}$ есть сумма растущей последовательности открытых множеств $Z_{\beta}, \beta<\omega_{1}$, гомеоморфных $\mathbb{R}^{4}$.

(4.9) ОСНОВНоЕ СВоЙСТВО ОТОБРАЖЕНИЯ $\pi_{0}$. Для всякого замкнутого множества $F \subset M^{4, C}$ множество $\pi_{0}\left(\mathrm{Cl}_{X}(F)\right) \backslash \pi_{0}^{\#}\left(\mathrm{Cl}_{X}(F)\right)$ счетно.

ДокАЗАТЕЛЬСтво. Заметим сначала, что для всякого $\beta<\omega_{1}$ и всякого множества $\Phi \subset X_{\beta}$ имеем

$$
\left|\pi_{0}^{\beta}(\Phi) \backslash\left(\pi_{0}^{\beta}\right)^{\#}(\Phi)\right| \leqslant \omega_{0},
$$

в силу свойства 6 спектра $S^{\varphi}$. Отметим также, что операщия малого образа функториальна, т.е.

$$
(g \circ f)^{\#}=g^{\#} \circ f^{\#} .
$$

Пусть теперь $\Psi \subset X-$ произвольное множество и $\beta<\omega_{1}$. Тогда

$$
\pi_{0}(\Psi) \backslash \pi_{0}^{\#}(\Psi)=Q_{1} \cup Q_{2},
$$

где $Q_{1}=\pi_{0}^{\beta}\left(\pi_{\beta}(\Psi) \backslash \pi_{\beta}^{\#}(\Psi)\right), Q_{2}=\pi_{0}^{\beta}\left(\pi_{\beta}^{\#}(\Psi)\right) \backslash\left(\pi_{0}^{\beta}\right)^{\#}\left(\pi_{\beta}^{\#}(\Psi)\right)$.

В самом деле, введем для краткости следуюшие обозначения:

$$
L=p_{0}(\Psi) \backslash \pi_{0}^{\#}(\Psi), \quad M=\pi_{\beta}(\Psi), \quad N=\pi_{\beta}^{\#}(\Psi) .
$$

В этих обозначениях $Q_{1}=\pi_{0}^{\beta}(M \backslash N), Q_{2}=\pi_{0}^{\beta}(N) \backslash\left(\pi_{0}^{\beta}\right)^{\#}(N)$. Имеем

$$
\begin{aligned}
L \stackrel{(4.11)}{=} \pi_{0}^{\beta}(M) \backslash\left(\pi_{0}^{\beta}\right)^{\#}(N) & =\pi_{0}^{\beta}((M \backslash N) \cup N) \backslash\left(\pi_{0}^{\beta}\right)^{\#}(N) \\
& =Q_{1} \cup \pi_{0}^{\beta}(N) \backslash\left(\pi_{0}^{\beta}\right)^{\#}(N)=Q_{1} \cup Q_{2},
\end{aligned}
$$

поскольку $A \cup B \backslash C=A \cup(B \backslash C)$, если $C \subset B$. 
Итак, равенство (4.12) проверено. Подставляя в него $\Psi=\mathrm{Cl}_{X}(F)$ и учитывая неравенство (4.10), получаем, что для доказательства нашего утверждения (4.9) достаточно найти $\beta<\omega_{1}$, для которого

$$
\left|\pi_{\beta}\left(\mathrm{Cl}_{X}(F)\right) \backslash \pi_{\beta}^{\#}\left(\mathrm{Cl}_{X}(F)\right)\right| \leqslant \omega_{0}
$$

Положим $R_{F}=\mathrm{Cl}_{X}(F) \cap S_{C}^{3}$. Если $R_{F}=\varnothing$, то $F=\mathrm{Cl}_{X}(F)$ является компактом, лежашим в некотором множестве $Z_{\beta}$ согласно (4.8). Тогда из непрерьвности спектра $S^{\varphi}$ и его свойства 8 следует, что отображение $\pi_{\beta} \mid \pi_{\beta}^{-1}\left(\mathrm{Cl}_{X}(F)\right)$ является гомеоморфизмом. Поэтому множество из (4.13) не просто счетно, а пусто.

Пусть теперь $R_{F}$ не пусто. Возьмем какое-нибудь счетное плотное в $R_{F}$ множество $\left\{b_{1}, \ldots, b_{i}, \ldots\right\}$. Согласно (4.6) для каждого $i$ существует последовательность $P_{i} \subset F$, сходящаяся к точке $b_{i}$. Положим $A^{\prime}=\bigcup_{i=1}^{\infty} P_{i}$. Из счетности множества $A^{\prime}$ и свойства (4.8) вытекает, что $A^{\prime} \subset Z_{\beta_{0}}$ для некоторого $\beta_{0}<\omega_{1}$. Множество $F \cap Z_{\beta_{0}}$ сепарабельно, поскольку $Z_{\beta_{0}}$ гомеоморфно $\mathbb{R}^{4}$. Следовательно, существует счетное множество $A \supset A^{\prime}$, плотное в $F \cap Z_{\beta_{0}}$. Как и всякое счетное подмножество множества $Z=Z_{\omega_{1}}=M^{4, C}$, множество $A$ имеет вид $A_{\delta}$ для некоторого $\delta<\omega_{1}$. Положим $\beta=\max \left\{\beta_{0}, \delta\right\}$. Из включения $A^{\prime} \subset A_{\delta}$ вытекает, что $R_{F} \subset \mathrm{Cl}_{X}\left(A_{\delta}\right)$.

Но $\pi_{\beta}\left(A_{\delta}\right)=A_{\delta}$, в силу свойств $(4.2), 1$ и $\delta \leqslant \beta$. Из того же свойства 1 получаем, что $\pi_{\beta}\left(R_{F}\right)=R_{F}$. Следовательно, всякая точка $s_{\gamma} \in R_{F}$ является предельной точкой множества $A_{\delta}$ в $X_{\beta}$. Тогда из свойства (4.7) вытекает, что

$$
\pi_{\beta}^{-1}\left(s_{\gamma}\right) \subset \mathrm{Cl}_{X}(F)
$$

для всех $s_{\gamma} \in R_{F}$ при $\gamma \geqslant \beta$.

В самом деле, если $\beta \leqslant \gamma$, то

$$
\pi_{\beta}^{-1}\left(s_{\gamma}\right)=\pi_{\gamma}^{-1}\left(s_{\gamma}\right)
$$

согласно свойству 1 спектра $S^{\varphi}$.

С другой стороны, если $\gamma<\beta$, то $\pi_{\gamma}^{-1}\left(s_{\gamma}\right)$ состоит из одной точки, в силу свойства 6 спектра $S^{\varphi}$. Поэтому из (4.14) получаем

$$
\pi_{\beta}^{-1}\left(R_{F}\right) \subset \mathrm{Cl}_{X}(F)
$$

В силу того же свойства 6 , если $x \in \pi_{\beta}\left(\mathrm{Cl}_{X}(F)\right) \backslash \pi_{\beta}^{\#}\left(\mathrm{Cl}_{X}(F)\right)$, то $x \notin Z_{\beta}$ и, значит, $x \in \pi_{\beta}\left(S_{C}^{3}\right)=S_{C}^{3}$. Поэтому с учетом свойства (4.15) для того, чтобы показать, что наше $\beta$ удовлетворяет неравенству (4.13), достаточно проверить счетность множества

$$
R \equiv S_{C}^{3} \cap \pi_{\beta}^{-1}\left(\pi_{\beta}\left(\mathrm{Cl}_{X}(F)\right)\right) \backslash R_{F}
$$

Но множество $R$ является локально компактньм сепарабельным метризуемым пространством. Предположив, что оно несчетно, находим такой несчетный компакт $D$, что

$$
D \subset R
$$


По определению топологии предела обратного спектра существуют такое $\beta_{1}, \beta \leqslant$ $\beta_{1}<\omega_{1}$, и такая окрестность $U$ множества $\pi_{\beta_{1}}(D)$ в компакте $X_{\beta_{1}}$, что

$$
\mathrm{Cl}_{X}\left(\pi_{\beta_{1}}^{-1}(U)\right) \cap R_{F}=\varnothing .
$$

Из (4.18) вытекает, что

$$
\pi_{\beta}^{-1}\left(s_{\gamma}\right) \cap F \neq \varnothing
$$

для любой точки $s_{\gamma} \in D$, так как $F=\mathrm{Cl}_{X}(F) \backslash R_{F}$.

Но согласно (4.15) при $\gamma \geqslant \beta_{1}$

$$
\pi_{\beta}^{-1}\left(s_{\gamma}\right)=\pi_{\beta_{1}}^{-1}\left(s_{\gamma}\right)=\pi_{\gamma}^{-1}\left(s_{\gamma}\right) .
$$

Значит, из (4.20) вытекает, что $\pi_{\beta_{1}}^{-1}\left(s_{\gamma}\right) \neq \varnothing$ для всех $s_{\gamma} \in D, \gamma \geqslant \beta$.

Возьмем по точке $z_{\gamma} \in \pi_{\beta_{1}}^{-1}\left(s_{\gamma}\right) \cap F$ для каждого $s_{\gamma} \in D$ при $\gamma \geqslant \omega_{1}$. Обозначим через $Z^{0}$ замькание в $X$ множества $Z^{1}=\left\{z_{\gamma}: s_{\gamma} \in D, \gamma \geqslant \beta_{1}\right\}$. Тогда

$$
Z^{0} \subset \mathrm{Cl}_{X}(F) \cap \mathrm{Cl}_{X}\left(\pi_{\beta_{1}}^{-1}(U)\right) .
$$

Поэтому, в силу (4.18), (4.19) и включения $Z^{1} \subset F$, множество $Z^{0}$ является компактом, лежащим в $M^{4, C}$. Значит, $Z^{0} \subset Z_{\beta_{2}}$ для некоторого $\beta_{2}, \beta_{1} \leqslant \beta_{2}<\omega_{1}$. Но это противоречит тому, что $Z^{0}$, в силу несчетности множества $D$, содержит точки $z_{\gamma}$ со сколь угодно большими номерами, поскольку $z_{\gamma} \notin Z_{\gamma^{\prime}}$ при $\gamma^{\prime}<\gamma$. Таким образом, счетность множества (4.17), а вместе с ней и основное свойство (4.9) отображения $\pi_{0}$ доказаны.

Для открытого множества $U \subset M^{4, C}$ через $\widetilde{U}$ мы будем обозначать такое наибольшее открытое в $X$ множество, что $\widetilde{U} \cap M^{4, C}=U$, т.е.

$$
\widetilde{U}=X \backslash \mathrm{Cl}_{X}\left(M^{4, C} \backslash U\right) .
$$

Из основного свойства отображения $\pi_{0}$ вытекает

(4.22) Множество $\pi_{0}(\widetilde{U}) \backslash \pi_{0}^{\#}(\widetilde{U})$ счетно для всякого открытого множества $U \subset M^{4, C}$.

В самом деле, положим $F=M^{4, C} \backslash U$. Тогда $\widetilde{U}=X \backslash \mathrm{Cl}_{X}(F)$ или $\mathrm{Cl}_{X}(F)=X \backslash \widetilde{U}$. Из (4.9) получаем, что множество

$$
\pi_{0}(X \backslash \widetilde{U}) \backslash \pi_{0}^{\#}(X \backslash \widetilde{U})
$$

счетно. Но

$$
\pi_{0}(X \backslash \widetilde{U})=\pi_{0}(X) \backslash \pi_{0}^{\#}(\widetilde{U})
$$

a

$$
\pi_{0}^{\#}(X \backslash \widetilde{U})=\pi_{0}(X) \backslash \pi_{0}(\widetilde{U}),
$$

откуда свойство (4.22) и вытекает.

Положим $\pi=\pi_{0} \mid M^{4, C}$. Тогда из (4.9) вытекает

(4.23) Множество $\mathrm{Cl}_{X_{0}}(\pi(F)) \backslash \pi \#(F)$ счетно для всякого замкнутого множества $F \subset M^{4, C}$. 
В самом деле, $\pi(F) \subset \pi_{0}\left(\mathrm{Cl}_{X}(F)\right)$ и, следовательно, $\mathrm{Cl}_{X_{0}}(\pi(F)) \subset \pi_{0}\left(\mathrm{Cl}_{X}(F)\right)$, в силу замкнутости отображения $\pi_{0}: X \rightarrow X_{0}$. С другой стороны, легко видеть, что $\pi_{0}^{\#}\left(\mathrm{Cl}_{X}(F)\right) \subset \pi^{\#}(F)$, откуда с учетом (4.9) свойство (4.23) и вытекает.

Двойственным образом свойство (4.23) отображения $\pi$ можно сформулировать так:

(4.24) Множество $\pi(U) \backslash \operatorname{Int}_{X_{0}}(\pi \#(U))$ счетно для всякого открытого множества $U \subset M^{4, C}$.

Отметим также, что из определения 1 проекций $\pi_{\gamma}^{\beta}$ вытекает равенство

$$
\pi_{0}\left(M^{4, C}\right)=X_{0}
$$

4.1. ЗАмЕчАниЕ. Легко видеть, что, повторяя практически дословно доказательство свойства (4.9) с заменой 0 на произвольный трансфинит $\eta<\omega_{1}$, получаем следуюшее свойство отображения $\pi_{\eta}: X \rightarrow X_{\eta}$.

$\left(4.9_{\eta}\right)$ Множество $\pi_{\eta}\left(\mathrm{Cl}_{X}(F)\right) \backslash \pi_{\eta}^{\#}\left(\mathrm{Cl}_{X}(F)\right)$ счетно для всякого замкнутого множества $F \subset M^{4, C}$.

Соответственно имеют место $\eta$-аналоги $\left(4.22_{\eta}\right),\left(4.23_{\eta}\right)$ и $\left(4.24_{\eta}\right)$ свойств $(4.22)$, (4.23) и (4.24). В то же время в равенстве (4.25) нельзя заменить 0 ни на какой больший трансфинит. Так, например, $\pi_{1}\left(M^{4, C}\right)=X_{1} \backslash\left\{s_{0}\right\}$.

4.2. ЗАмечАниЕ. Дадим еще один комментарий к свойству (4.9). Поскольку $\pi_{0} \mid \pi_{0}^{-1}\left(Z_{0}\right)$ - гомеоморфизм, имеем

$$
\pi_{0}\left(\mathrm{Cl}_{X}(F)\right) \backslash \pi_{0}^{\#}\left(\mathrm{Cl}_{X}(F)\right) \subset S_{C}^{3}
$$

Значит, существует такой счетный трансфинит $\gamma$, что

$$
\pi_{0}\left(\mathrm{Cl}_{X}(F)\right) \backslash \pi_{0}^{\#}\left(\mathrm{Cl}_{X}(F)\right) \subset\left\{s_{\gamma^{\prime}}: \gamma^{\prime}<\gamma\right\}
$$

Отсюда вытекает свойство

(4.27) Для любого замкнутого множества $F \subset M^{4, C}$ существует такое $\beta<\omega_{1}$, что

$$
\mathrm{Cl}_{X}(F)=\pi_{\beta}^{-1}\left(\pi_{\beta}\left(\mathrm{Cl}_{X}(F)\right)\right) .
$$

В качестве такого $\beta$ можно взять любое $\beta \geqslant \gamma$, где $\gamma$ удовлетворяет условию (4.26). В самом деле, пусть $x \in \pi_{\beta}\left(\mathrm{Cl}_{X}(F)\right)$. Если $x \in Z_{\beta}$, то $\pi_{\beta}^{-1}(x)=x \in \mathrm{Cl}_{X}(F)$. Если же $x=s_{\beta^{\prime}}$ то при $\beta^{\prime}<\gamma \leqslant \beta$ имеем $\pi_{\beta}^{-1}(x)=\pi_{\gamma}^{-1}(x)=x$ согласно свойству 6 спектра $S^{\varphi}$. А при $\beta^{\prime} \geqslant \gamma$ имеем $s_{\beta^{\prime}} \in \pi_{0}^{\#}\left(\mathrm{Cl}_{X}(F)\right)$ согласно (4.26). Поэтому $\pi_{\beta}^{-1}\left(s_{\beta^{\prime}}\right) \subset \pi_{0}^{-1}\left(s_{\beta^{\prime}}\right) \subset \mathrm{Cl}_{X}(F)$.

Соответствующим образом можно переформулировать и свойства $(4.22),(4.23)$ и (4.24). 
4.3. ЗАмЕчАНИЕ. Легко убедиться в том, что в формулировке свойства (4.9) мы можем предполагать, что $F$ является замкнутым множеством в $\pi^{-1}(E)$, где $E$ - некоторое замкнутое подмножество пространства $X_{0}$. При этом, естественно, $\mathrm{Cl}_{X}(F)$ заменяется на $\mathrm{Cl}_{\pi_{0}^{-1}(E)}(F)$. То же самое относится и к вытекающему из (4.9) свойству (4.23). Эти новые формулировки фактически совпадают со старыми.

Новую информацию мы получаем, лиш переходя к дополнениям. Так, свойство (4.22) трансформируется в свойство

$\left(4.22_{E}\right)$ Пусть $E$ замкнуто в $X_{0}$. Тогда для всякого открытого в $\pi^{-1}(E)$ множества $U$ счетным является множество $\pi_{0}(\widetilde{U}) \backslash \pi_{0}^{\#}(\widetilde{U})$, где $\widetilde{U}-$ наибольшее в $\pi_{0}^{-1}(E)$ множество, удовлетворяющее условию $U=\widetilde{U} \cap \pi^{-1}(E)$.

Отметим еще одно свойство, которое практически очевидно.

(4.28) Многообразие $M^{4, C}$ плотно в компакте $X$.

В самом деле, сфера $S^{3}$ нигде не плотна в $B^{4}$. В то же время $S^{3}=\varphi^{-1}\left(S_{C}^{3}\right)$. Следовательно, компакт $S_{C}^{3}$ нигде не плотен в $B_{C}^{4}$. Тогда $X \backslash M^{4, C}=S_{C}^{3}$ является подмножеством предела спектра $S^{\varphi}$, образ которого относительно любой сквозной проекции $\pi_{\beta}$ нигде не плотен в $X_{\beta}$ согласно свойствам 3,4 и 9 спектра $S^{\varphi}$. Отсюда и вытекает, что множество $X \backslash M^{4, C}$ нигде не плотно в $X$.

(4.29) Многообразие $M^{4, C}$ совершенно нормально и наследственно сепарабельно.

Нормальность $M^{4, C}$ доказывается точно так же, как и нормальность многообразия $M^{n, m}$ (см. [37; предложение 2.1]). При этом используются нормализующая лемма (см. [5; гл. $1, \S 5$, лемма 2]) и свойство (4.23) отображения $\pi$. Совершенная нормальность многообразия $M^{4, C}$ вытекает из того, что всякое его открытое подмножество

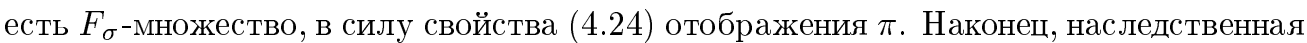
сепарабельность совершенно нормального многообразия вытекает из его сепарабельности по теореме 1.14. Сепарабельность же многообразия $M^{4, C}$ вытекает из того, что, как легко видеть, множество $Z_{0}=B_{C}^{4} \backslash S_{C}^{3}$ плотно в $Z$. В самом деле, $Z_{0}$ плотно в $Z_{\beta}$ для всякого $\beta<\omega_{1}$, поскольку по свойству 6 спектра $S^{\varphi}$ разность $Z_{\beta} \backslash Z_{0}$ является суммой счетного числа замкнутых и не более чем двумерных и, следовательно, нигде не плотных подмножеств топологически полного пространства $Z_{\beta}$. Но если $Z_{0}$ плотно в каждом $Z_{\beta}$, то $Z_{0}$ плотно и в объединении $\bigcup\left\{Z_{\beta}: \beta<\omega_{1}\right\}=Z=M^{4, C}$.

\section{$\S 5$. Основные результаты}

В этом параграфее мы будем предполагать, что

$$
\operatorname{dim} C \geqslant 5
$$

Начнем с размерности компакта $X$. Имеет место равенство

$$
\operatorname{dim} X=\operatorname{dim} C \text {. }
$$

В самом деле, $\operatorname{dim} X \geqslant \operatorname{dim} C$, поскольку $X \supset C . \mathrm{C}$ другой стороны, $X$ есть предел обратного спектра $S^{\varphi}$, состоящего из компактов $X_{\beta}$, гомеоморфных компакту $B_{C}^{4}$ согласно свойству 3. Поэтому $\operatorname{dim} X \leqslant \operatorname{dim} B_{C}^{4}$. Но из (5.1) вытекает, что $\operatorname{dim} B_{C}^{4}=\operatorname{dim} C$, поскольку $B_{C}^{4} \backslash C$ гомеоморфино открытому подмножеству шара $B^{4}$. 
5.1. ТЕОРемА. Для всякого замкнутого множества $F \subset M^{4, C}$ имеем

$$
\operatorname{dim} \mathrm{Cl}_{X}(F)-1 \leqslant \operatorname{dim} F \leqslant \operatorname{dim} \mathrm{Cl}_{X}(F) .
$$

$B$ частности, если $\operatorname{dim} \mathrm{Cl}_{X}(F)=\infty$, то $\operatorname{dim} F=\infty$.

ДоказАТЕльство. Пусть $\operatorname{dim} \mathrm{Cl}_{X}(F)=m$. Проверим сначала правое неравенство. Пусть $u_{0}=\left\{U_{1}^{0}, \ldots, U_{k}^{0}\right\}$ - произвольное конечное открытое покрытие множества $F$. Сушествуют такие открытые в $M^{4, C}$ множества $U_{i}$, что $U_{i} \cap F=U_{i}^{0}$ и семейство $u=\left\{U_{1} \ldots, U_{k}\right\}$ является покрытием многообразия $M^{4, C}$. В самом деле, можно положить, например, $U_{i}=U_{i}^{0} \cup\left(M^{4, C} \backslash F\right)$. Положим $\widetilde{u}=\left\{\widetilde{U}_{1}, \ldots, \widetilde{U}_{k}\right\}$ и $B=X \backslash \bigcup \widetilde{u}$. Покажем, что

(5.4) Множество $B$ счетно.

Из того, что $u$ является покрытием многообразия $M^{4, C}$, вытекает справедливость включения $B \subset S_{C}^{3}$. Но уже из определения 1 проекций нетопологизированного спектра $S^{\varphi}$ вытекает, что все отображения $\pi_{\gamma}^{\beta} \mid S_{C}^{3}$ взаимно однозначны. Следовательно, этим же свойством обладает и отображение $\pi_{0} \mid S_{C}^{3}$. Значит, $|B|=\left|\pi_{0}(B)\right|$. Далее, имеет место включение

$$
\pi_{0}(B) \subset \bigcup_{i=1}^{k} \pi_{0}\left(\widetilde{U}_{i}\right) \backslash \bigcup_{i=1}^{k} \pi_{0}^{\#}\left(\widetilde{U}_{i}\right)
$$

В самом деле, очевидно, что

$$
\pi_{0}(B) \cap\left(\bigcup_{i=1}^{k} \pi_{0}^{\#}\left(\widetilde{U}_{i}\right)\right)=\varnothing
$$

С другой стороны,

$$
\bigcup_{i=1}^{k} \pi_{0}\left(\widetilde{U}_{i}\right)=\pi_{0}\left(\bigcup_{i=1}^{k} \widetilde{U}_{i}\right) \supset \pi_{0}\left(\bigcup_{i=1}^{k} U_{i}\right)=\pi_{0}\left(M^{4, C}\right) \stackrel{(4.25)}{=} X_{0} \supset \pi_{0}(B) .
$$

Из (5.5), (4.22) и (5.20) (см. ниже) вытекает счетность множества $\pi_{0}(B)$, а вместе с этим и счетность множества $B$.

Положим

$$
B_{F}=B \cap \mathrm{Cl}_{X}(F), \quad \widetilde{U}_{i}^{0}=\widetilde{U}_{i} \cap \mathrm{Cl}_{X}(F)
$$

и

$$
\widetilde{u}_{0}=\left\{\widetilde{U}_{1}^{0}, \ldots, \widetilde{U}_{k}^{0}\right\}
$$

Тогда $\mathrm{Cl}_{X}(F) \backslash B_{F}=\bigcup \widetilde{u}_{0}$. Но $B_{F}$ является счетньм компактом, лежашим в компакте $\mathrm{Cl}_{X}(F)$, которьй согласно (4.6) удовлетворяет первой аксиоме счетности. Значит, $B_{F}$ есть $G_{\delta}$-множество в компакте $\mathrm{Cl}_{X}(F)$ по предложению 1.7. Тогда $\bigcup \widetilde{u}_{0}$ есть $F_{\sigma}$-множество в компакте $\mathrm{Cl}_{X}(F)$. Следовательно, $\operatorname{dim}\left(\bigcup \widetilde{u}_{0}\right) \leqslant m$ в силу теоремы счетной суммы для лебеговой размерности. Значит, существует открытое покрытие $v$ множества $\mathrm{Cl}_{X}(F) \backslash B_{F}$, вписанное в покрытие $\widetilde{u}_{0}$ и имеющее кратность $\leqslant m+1$. Тогда $w=v \mid F$ является открытьм покрытием множества $F$, вписанным в $u_{0}$ и имеющим кратность $\leqslant m+1$. Таким образом, $\operatorname{dim} F \leqslant \operatorname{dim} \mathrm{Cl}_{X}(F)$. 
Теперь проверим левое неравенство из (5.3). Для этого докажем сначала, что

$$
\operatorname{dim} R_{F}-1 \leqslant \operatorname{dim} F
$$

где $R_{F}=\mathrm{Cl}_{X}(F) \cap S_{C}^{3}$. Пусть $\operatorname{dim} R_{F}=n$. Тогда согласно предложению 1.2 сушествуют такие пары $\left(A_{i}^{1}, A_{i}^{2}\right), 1 \leqslant i \leqslant n-1$, непересекаюшихся замкнутых подмножеств компакта $\pi_{0}\left(R_{F}\right)=R_{F}$, что

$$
\operatorname{dim}\left(\bigcap\left\{B_{i}: 1 \leqslant i \leqslant n-1\right\}\right) \geqslant 1
$$

для любых перегородок $B_{i}$ в пространстве $\pi_{0}\left(R_{F}\right)$ между множествами $A_{i}^{1}$ и $A_{i}^{2}$. Пусть $U_{i}^{j}$ - такие окрестности множеств $A_{i}^{j}$ в компакте $\pi_{0}\left(\mathrm{Cl}_{X}(F)\right)$, что $\mathrm{Cl}\left(U_{i}^{1}\right) \cap \mathrm{Cl}\left(U_{i}^{2}\right)=\varnothing$ для всех $i$. Положим

$$
F_{i}^{j}=F \cap \pi_{0}^{-1}\left(\mathrm{Cl}\left(U_{i}^{j}\right)\right) .
$$

Для того чтобы проверить неравенство $n-1 \leqslant \operatorname{dim} F$, достаточно согласно теореме 1.1 показать, что

$$
\bigcap\left\{C_{i}: 1 \leqslant i \leqslant n-1\right\} \neq \varnothing
$$

для любых перегородок $C_{i}$ в $F$ между множествами $F_{i}^{1}$ и $F_{i}^{2}$. Пусть $F \backslash C_{i}=V_{i}^{1} \cup V_{i}^{2}$, где $V_{i}^{1}$ и $V_{i}^{2}$ - непересекаюшиеся окрестности множеств $F_{i}^{1}$ и $F_{i}^{2}$ в $F$. Положим

$$
D_{i}=\mathrm{Cl}_{X}(F) \backslash \widetilde{V}_{i}^{1} \cup \widetilde{V}_{i}^{2}
$$

где $\widetilde{V}_{i}^{j}$ - наибольшее открытое в $\mathrm{Cl}_{X}(F)$ множество со свойством $\tilde{V}_{i}^{j} \cap F=V_{i}^{j}$.

Рассмотрим сначала случай

$$
\mathrm{Cl}_{X}(F)=\pi_{0}^{-1}\left(\pi_{0}\left(\mathrm{Cl}_{X}(F)\right)\right)
$$

Ясно, что

$$
\pi^{-1}(E)=F \cap \pi_{0}^{-1}(E)
$$

для любого множества $E \subset \pi_{0}\left(\mathrm{Cl}_{X}(F)\right)$. В частности,

$$
F=\pi^{-1}\left(\pi_{0}\left(\mathrm{Cl}_{X}(F)\right)\right)
$$

и

$$
\pi^{-1}\left(U_{i}^{j}\right)=F \cap \pi_{0}^{-1}\left(U_{i}^{j}\right) .
$$

Следовательно, $\pi_{0}^{-1}\left(U_{i}^{j}\right)$ является открытьм в $\mathrm{Cl}_{X}(F)$ множеством, след которого на $F$ равен $\pi^{-1}\left(U_{i}^{j}\right)$. Значит,

$$
\pi_{0}^{-1}\left(U_{i}^{j}\right) \subset \widetilde{\pi^{-1}\left(U_{i}^{j}\right)} .
$$

Из (5.8) вытекает, что $\pi^{-1}\left(U_{i}^{j}\right) \subset F_{i}^{j}$. Следовательно,

$$
\pi^{-1}\left(U_{i}^{j}\right) \subset V_{i}^{j}
$$


откуда

$$
\widetilde{\pi^{-1}\left(U_{i}^{j}\right)} \subset \widetilde{V}_{i}^{j}
$$

Поэтому из (5.15) получаем

$$
\pi_{0}^{-1}\left(A_{i}^{j}\right) \subset \pi_{0}^{-1}\left(U_{i}^{j}\right) \subset \widetilde{V}_{i}^{j} .
$$

Из (5.16) вытекает, что $D_{i}$ является перегородкой в компакте $\mathrm{Cl}_{X}(F)$ между множествами $\pi_{0}^{-1}\left(A_{i}^{1}\right)$ и $\pi_{0}^{-1}\left(A_{i}^{2}\right)$. Но, в силу (5.11), отображение $\pi_{0} \mid \mathrm{Cl}_{X}(F)$ монотонно как ограничение монотонного отображения $\pi_{0}$ на полньй прообраз. Поэтому по предложению 1.3 множество $\pi_{0}\left(D_{i}\right)$ является перегородкой в компакте $\pi_{0}\left(\mathrm{Cl}_{X}(F)\right)$ между множествами $A_{i}^{1}$ и $A_{i}^{2}$. Следовательно, из (5.7) вытекает, что

$$
\operatorname{dim}\left(\bigcap\left\{\pi_{0}\left(D_{i}\right): 1 \leqslant i \leqslant n-1\right\}\right) \geqslant 1 .
$$

Таким образом, условие (5.9) будет вьполняться, если мы покажем, что

$$
\left|\bigcap_{i} \pi_{0}\left(D_{i}\right) \backslash \bigcap_{i} \pi^{\#}\left(C_{i}\right)\right| \leqslant \omega_{0} .
$$

В самом деле, из (5.17) и (5.18) вытекает, что множество $\bigcap_{i} \pi \#\left(C_{i}\right)$ несчетно, поскольку получается вычитанием из несчетного множества $\bigcap_{i} \pi_{0}\left(D_{i}\right)$ некоторого счетного множества. Тогда несчетны множество $\pi^{-1}\left(\bigcap_{i} \pi^{\#}\left(C_{i}\right)\right)$ и содержащее его множество $\bigcap_{i} C_{i}$.

Для проверки свойства (5.18) нам потребуются два элементарных теоретико-множественных факта:

$$
\begin{aligned}
& \bigcap_{\alpha} P_{\alpha} \backslash \bigcap_{\alpha} Q_{\alpha} \subset \bigcup_{\alpha}\left(P_{\alpha} \backslash Q_{\alpha}\right), \\
& \bigcup_{\alpha} P_{\alpha} \backslash \bigcup_{\alpha} Q_{\alpha} \subset \bigcup_{\alpha}\left(P_{\alpha} \backslash Q_{\alpha}\right) .
\end{aligned}
$$

Следовательно, для доказательства утверждения (5.18) достаточно (в силу (5.19)) показать, что

$$
\left|\pi_{0}\left(D_{i}\right) \backslash \pi^{\#}\left(C_{i}\right)\right| \leqslant \omega_{0} .
$$

А для этого согласно свойству (4.20) отображения $\pi$ достаточно проверить, что

$$
\left|\pi_{0}\left(D_{i}\right) \backslash \pi\left(C_{i}\right)\right| \leqslant \omega_{0} .
$$

Из равенства $F \backslash C_{i}=V_{i}^{1} \cup V_{i}^{2}$ получаем

$$
\pi\left(F \backslash C_{i}\right)=\pi\left(V_{i}^{1}\right) \cup \pi\left(V_{i}^{2}\right)
$$

и, значит,

$$
\pi(F) \backslash \pi\left(C_{i}\right)=\pi\left(V_{i}^{1}\right) \cup \pi\left(V_{i}^{2}\right) .
$$


Но $\pi(F)=\pi_{0}\left(\mathrm{Cl}_{X}(F)\right)$ согласно (5.13). Следовательно,

$$
\pi_{0}\left(\mathrm{Cl}_{X}(F)\right) \backslash \pi\left(C_{i}\right)=\pi\left(V_{i}^{1}\right) \cup \pi\left(V_{i}^{2}\right) .
$$

Из равенства (5.11) получаем

$$
\pi_{0}\left(D_{i}\right)=\pi_{0}\left(\mathrm{Cl}_{X}(F)\right) \backslash \pi_{0}^{\#}\left(\tilde{V}_{i}^{1} \cup \tilde{V}_{i}^{2}\right) .
$$

Но из монотонности отображения $\pi_{0}$ и дизъюнктности открытых множеств $\widetilde{V}_{i}^{1}$ и $\widetilde{V}_{i}^{2}$ вытекает, что

$$
\pi_{0}^{\#}\left(\widetilde{V}_{i}^{1} \cup \widetilde{V}_{i}^{2}\right)=\pi_{0}^{\#}\left(\widetilde{V}_{i}^{1}\right) \cup \pi_{0}^{\#}\left(\widetilde{V}_{i}^{2}\right) .
$$

Значит,

$$
\pi_{0}\left(D_{i}\right)=\pi_{0}\left(\mathrm{Cl}_{X}(F)\right) \backslash \pi_{0}^{\#}\left(\widetilde{V}_{i}^{1}\right) \cup \pi_{0}^{\#}\left(\widetilde{V}_{i}^{2}\right) .
$$

Тогда

$$
\begin{array}{r}
\pi_{0}\left(D_{i}\right) \backslash \pi\left(C_{i}\right) \stackrel{(5.23),(5.24)}{\subset} \pi\left(V_{i}^{1}\right) \cup \pi\left(V_{i}^{2}\right) \backslash \pi_{0}^{\#}\left(\widetilde{V}_{i}^{1}\right) \cup \pi_{0}^{\#}\left(\widetilde{V}_{i}^{2}\right) \\
\stackrel{(5.20)}{\subset}\left(\pi\left(V_{i}^{1}\right) \backslash \pi_{0}^{\#}\left(\widetilde{V}_{i}^{1}\right)\right) \cup\left(\pi\left(V_{i}^{2}\right) \backslash \pi_{0}^{\#}\left(\widetilde{V}_{i}^{2}\right)\right) .
\end{array}
$$

Но последнее множество содержится в множестве

$$
\left(\pi_{0}\left(\widetilde{V}_{i}^{1}\right) \backslash \pi_{0}^{\#}\left(\widetilde{V}_{i}^{1}\right)\right) \cup\left(\pi_{0}\left(\widetilde{V}_{i}^{2}\right) \backslash \pi_{0}^{\#}\left(\widetilde{V}_{i}^{2}\right)\right),
$$

которое счетно по свойству $\left(4.22_{E}\right)$ (см. замечание 4.3$)$ для $E=\pi_{0}\left(\mathrm{Cl}_{X}(F)\right)$. Утверждение (5.21), а вместе с ним и утверждения (5.18) и (5.9) доказаны. Следовательно, доказано неравенство (5.6) при выполнении равенства (5.11).

В общем случае для доказательства неравенства (5.6) вместо (5.11) надо воспользоваться свойством (4.27) из замечания 4.2 и повторить предыдущее доказательство, заменяя 0 на $\beta$, а свойства $(4.9),(4.22),(4.23)$ и $(4.24)$ на свойства $\left(4.9_{\beta}\right),\left(4.22_{\beta}\right)$, $\left(4.23_{\beta}\right)$ и $\left(4.24_{\beta}\right)$ (см. замечание 4.1$)$.

Теперь теорема 5.1 доказывается в несколько слов. Очевидно, что $\operatorname{dim} K \leqslant \operatorname{dim} F$ для всякого компакта $K \subset F$. Поэтому из теоремы Даукера (теорема 1.5) вытекает

$$
\operatorname{dim}\left(\mathrm{Cl}_{X}(F)\right) \leqslant \max \left\{\operatorname{dim} R_{F}, \operatorname{dim} F\right\} .
$$

Поэтому применение неравенства (5.6) завершает доказательство теоремы 5.1.

Из (5.1), (5.2) и теоремы 5.1 вытекает

5.2. СлеДСтвиЕ. $\operatorname{dim} C-1 \leqslant \operatorname{dim} M^{4, C} \leqslant \operatorname{dim} C$.

В частности, для $C=I^{m}$, получаем

5.3. СлеДСтвИЕ. Если $m \geqslant 5, m o m-1 \leqslant \operatorname{dim} M^{4, I^{m}} \leqslant m$.

Непосредственно из теоремы 5.1 вытекает

5.4. СледСтвиЕ. Ecлu $\operatorname{dim} C=\infty, m o \operatorname{dim} M^{4, C}=\infty$. 
5.5. ТеОРемА. В предположсении континуум-гипотезы существует бесконечномерное, дифференцируемое, совершенно нормальное, наследственно сепарабельное 4-многообразие $M_{1}^{4}$, для каждого замкнутого подмножества $F$ которого мы имеем: либо $\operatorname{dim} F \leqslant 4$, либо $\operatorname{dim} F=\infty$.

ДокАЗАТЕЛЬСтво. Положим $M_{1}^{4}=M^{4, C}$, где $C$ - компакт Хендерсона, не имеюший замкнутых подмножеств положительной размерности [48]. Из следствия 5.4 вытекает, что $\operatorname{dim} M_{1}^{4}=\infty$. Пусть $F$ замкнуто в $M_{1}^{4}$ и $\operatorname{dim} F \geqslant 5$. Тогда $\operatorname{dim} \mathrm{Cl}_{X}(F) \geqslant 5$ по теореме 5.1. Следовательно, $\operatorname{dim} R_{F} \geqslant 5$ согласно теореме 1.5, так как $\operatorname{dim} K \leqslant 4$ для всякого замкнутого в $X$ множества $K \subset F$. Поскольку $\operatorname{dim}\left(S_{C}^{3} \backslash C\right)=3$, компакт $R_{F}$ пересекает компакт Хендерсона $C$ по множеству положительной размерности. Значит, $\operatorname{dim} R_{F}=\infty$, откуда $\operatorname{dim} \mathrm{Cl}_{X}(F)=\infty$, а по теореме 5.1 и $\operatorname{dim} F=\infty$. Теорема 5.5 доказана.

5.6. Теорема. В предположсении континуум-гипотезы существует бесконечномерное, дифференцируемое, совершенно нормальное, наследственно сепарабельное 4-многообразие $M_{1}^{4}$, для каждого открытого подмножества $U$ которого мы имеем: либо $\operatorname{dim} U=4, л и б о ~ \operatorname{dim} U=\infty$.

5.7. ТеоремА. В предположсении континуум-гипотезы существуют такое бесконечномерное, дифференцируемое, совершенно нормальное, наследственно сепарабельное 4-многообразие $M_{2}^{4}$ и такие его открытые подмножества $U_{i}, i=$ $1, \ldots, n, \ldots$, ито

a) $\operatorname{dim} U_{i} \neq \operatorname{dim} U_{j}$ npu $i \neq j$.

При этом мьи можем предполагать, что

б) либо $\mathrm{Cl}\left(U_{i}\right) \cap \mathrm{Cl}\left(U_{j}\right)=\varnothing$, если $i \neq j$,

в) либо $\bigcap\left\{U_{i}: i=1, \ldots, n, \ldots\right\} \neq \varnothing$.

ДокАЗАТЕльство. Многообразие $M_{2}^{4}$ также будет многообразием вида $M^{4, C}$. В качестве $C$ возьмем александровскую компактификацию дискретной суммы четномерных кубов $I^{2 i}, i \geqslant 3$. Сушествуют такие функционально открытые окрестности $V_{i}$ компактов $I^{2 i}, i \geqslant 3$, в компакте $X$, что

$$
\mathrm{Cl}_{X}\left(V_{i}\right) \cap \mathrm{Cl}_{X}\left(V_{j}\right)=\varnothing, \text { если } i \neq j .
$$

Для реализации свойства б) положим

$$
U_{i}=V_{i+2} \cap M_{2}^{4}
$$

Если же мы хотим получить свойство в), положим

$$
U_{i}=\left(V_{i+2} \cap M_{2}^{4}\right) \cup Z_{0} .
$$

Докажем, что

$$
2 i-1 \leqslant \operatorname{dim}\left(V_{i} \cap M_{2}^{4}\right) \leqslant 2 i .
$$

Из (5.28) очевидно будет вытекать свойство а) в случае (5.26). То же самое будет и в случае (5.27), поскольку

$$
\operatorname{dim}\left(\left(V_{j} \cap M_{2}^{4}\right) \cup Z_{0}\right)=\operatorname{dim}\left(V_{j} \cap M_{2}^{4}\right),
$$


в силу того, что $\operatorname{dim}\left(V_{j} \cap M_{2}^{4}\right) \geqslant 5, \operatorname{adim} Z_{0}=4$ и $Z_{0}$ является суммой счетного числа своих компактных подмножеств.

Итак, доказываем свойство (5.28). В силу функциональной открытости множества $V_{i}$ сушествуют такие окрестности $V_{i}^{k}$ куба $I^{2 i} \subset X$, что $\mathrm{Cl}\left(V_{i}^{k}\right) \subset V_{i}^{k+1}$ и $V_{i}=$ $\bigcup_{k=1}^{\infty} V_{i}^{k}$. Тогда

$$
V_{i} \cap M_{2}^{4}=\bigcup_{k=1}^{\infty}\left(\mathrm{Cl}\left(V_{i}^{k}\right) \cap M_{2}^{4}\right) .
$$

Поэтому для выполнения свойства (5.28), в силу теоремы счетной суммы для лебеговой размерности, достаточно проверить, что

$$
2 i-1 \leqslant \operatorname{dim}\left(\mathrm{Cl}\left(V_{i}^{k}\right) \cap M_{2}^{4}\right) \leqslant 2 i .
$$

Обозначим $\mathrm{Cl}\left(V_{i}^{k}\right) \cap M_{2}^{4}$ через $F$ и покажем, что

$$
\mathrm{Cl}_{X}(F)=\mathrm{Cl}\left(V_{i}^{k}\right)
$$

Включение $\mathrm{Cl}_{X}(F) \subset \mathrm{Cl}\left(V_{i}^{k}\right)$ очевидно. С другой стороны, из свойства (4.28) вытекает, что $V_{i}^{k} \cap M_{2}^{4}$ плотно в $V_{i}^{k}$. Но отсюда получаем, что $F$ плотно в $\mathrm{Cl}\left(V_{i}^{k}\right)$ и, значит, $\mathrm{Cl}_{X}(F)=\mathrm{Cl}\left(V_{i}^{k}\right)$.

Из теоремы 5.1 и равенства (5.30) получаем

$$
\operatorname{dim} \operatorname{Cl}\left(V_{i}^{k}\right)-1 \leqslant \operatorname{dim} F \leqslant \operatorname{dim} \operatorname{Cl}\left(V_{i}^{k}\right) .
$$

Поэтому свойство (5.29) будет доказано, как только мы докажем, что

$$
\operatorname{dim} \mathrm{Cl}\left(V_{i}^{k}\right)=2 i
$$

Но $\operatorname{dim} K \leqslant 4$ для всякого компакта $K \subset F=\mathrm{Cl}\left(V_{i}^{k}\right) \cap M_{2}^{4}$. Поэтому по уже упоминавшейся теореме Даукера 1.5 для вьполнения равенства (5.32) достаточно, чтобы

$$
\operatorname{dim}\left(\mathrm{Cl}\left(V_{i}^{k}\right) \cap S_{C}^{3}\right)=2 i .
$$

$\mathrm{Ho} \mathrm{Cl}\left(V_{i}^{k}\right) \cap S_{C}^{3} \supset I^{2 i}$, а разность $\mathrm{Cl}\left(V_{i}^{k}\right) \cap S_{C}^{3} \backslash I^{2 i}$ гомеоморфна подмножеству сферы $S^{3}$, откуда равенство (5.33) и вытекает. Теорема 5.6 доказана.

5.8. ЗАмечАниЕ. Поскольку в основной лемме 1.15 нет ограничений сверху на размерность $n$, конструкцию из $\$ 4$ и основные результаты из настоящего параграфа можно распространить с $n=4$ на любое целое $n \geqslant 4$.

В связи с теоремой 5.5 возникает

5.9. Вопрос. Существуют ли конечномерные многообразия без промежуточных размерностей? В частности, существует ли такое конечномерное 4 -многообразие $M^{4}$ размерности $\operatorname{dim} M^{4}=m, 6 \leqslant m<\infty$, что $\operatorname{dim} F \leqslant 4$ или $\operatorname{dim} F=m$ для всякого замкнутого множества $F \subset M^{4}$ ? 
5.10. ЗАмЕЧАниЕ. По поводу вопроса 5.9 стоит отметить, что в аксиоматике ZFC существуют конечномерные компакты без промежуточных размерностей [29]. В континуум-гипотезе сушествуют и совершенно нормальные компакты без промежуточных размерностей (см. [98], [33]).

В связи с теоремой 5.7 возникает

5.11. Вопрос. Существует ли в предположении континуум-гипотезы 4-многообразие, лебеговы размерности открытых подмножеств которого принимают все значения $n \geqslant 4$ ?

Ответ на вопрос 5.11 положителен, если вместо континуум-гипотезы предположить принцип Йенсена $\diamond$. Это вытекает из того, что для любого счетного множества $\mathscr{L}$ размерностных типов $[L]$ метризуемых компактов при $[L] \geqslant\left[S^{4}\right]$ сушествует дифференшируемое, счетно компактное, совершенно нормальное, наследственно сепарабельное 4-многообразие $M^{4}$, содержашее открытые подмножества $U_{L},[L] \in \mathscr{L}$, размерности продолжения $e-\operatorname{dim} U_{L}=[L]$ (см. [17; теорема 5.2]).

В заключение напомним один из основных вопросов о размерности неметризуемых многообразий.

5.12. Вопрос. Существует ли "наивньй" пример многообразия с несовпадаюшими размерностями?

Фактически, это - целая группа вопросов, зависящая от рассматриваемых размерностных инвариантов и свойств топологической и дифференщиальной структур. Ясно, что такое многообразие не может быть совершенно нормальным, в силу отмеченной во введении теоремы М. Рудин [97]. А по теореме Сентмиклоши [126] о финальной компактности (в предположении $\mathrm{MA}+\neg \mathrm{CH}$ ) всякого наследственно сепарабельного локально компактного пространства оно не может быть наследственно сепарабельным.

\section{СПИСОК ЛИТЕРАТУРЫ}

[1] С. М. Агеев, С. А. Богатый. О препятствиях к продолжению частичных отображений // Матем. заметки. 1997. Т. 62. №6. С. 803-812.

[2] П. С. Александров. О размерности бикомпактных пространств // Докл. АН СССР. 1940. T. 26. C. $627-630$.

[3] P. Alexandroff. On the dimension of normal spaces // Proc. Roy. Soc. London Ser. A. 1947. V. 189. P. 11-39.

[4] П. С. Александров. Предисловие к рус. пер. кн.: В. Гуревич, Г. Волмэн. Теория размерности. М.: ИЛ, 1948.

[5] П. С. Александров, Б. А. Пасынков. Введение в теорию размерности. М.: Наука, 1973.

[6] R. D. Anderson. Monotone interior dimension-raising mappings // Duke Math. J. 1952. V. 19. P. $359-366$.

[7] А. В. Архангельский. О факторизации отображений по весу и размерности // Докл. AH CCCP. 1967. T. 174. C. 1243-1246.

[8] R.H. Bing. Higher-dimensional hereditarily indecomposable continua // Trans. Amer. Math. Soc. 1951. V. 71. № 2. P. 267-273.

[9] С. А. Богатый. О метрических ретрактах // Докл. АН СССР. 1972. Т. 204.№ 3. C. $522-524$.

[10] С. А. Богатый. Характеристики топологической и равномерной размерности в терминах колец непрерьвных функций // Сиб. матем. журн. 1973. Т. 14. № 2. С. 289-299. 
[11] L. E. J.Brouwer. Beweis der Invarianz der Dimensionzahl // Math. Ann. 1911. V. 70. P. 161-165.

[12] L. E. J. Brouwer. Über den naturlichen Dimensionsbegriff // J. Reine Angew. Math. 1913. V. 142. P. 145-152.

[13] M. Brown. Some applications of an approximation theorem for inverse limits // Proc. Amer. Math. Soc. 1960. V. 11. № 3. P. 478-483.

[14] G. Cantor. Über unendlichelineare Punktmannichfaltigkeiten // Math. Ann. 1883. V. 21. P. $545-586$.

[15] E. Čech. Sur la dimension des espaces parfaitement normaux // Bull. Int. Acad. Tcheque Sci. 1932. V. 33. P. 38-55.

[16] А. Ч. Чигогидзе. О бесконечномерных бикомпактах // Сиб. матем. журн. 1982. Т. 23. C. $157-164$.

[17] A. Ch. Chigogidze, V. V. Fedorchuk. On some dimensional properties of 4-manifolds // Topology Appl. 2000. V. 107. № 1-2. P. 67-78.

[18] E. K. van Douwen. Mild infinite dimensionality of $\beta X$ and $\beta X-X$ for metrizable $X / /$ Topology Appl. 1993. V. 51. № 2. P. 93-108. (Препринт распространен в 1979 г.)

[19] C. H. Dowker. Mapping theorems for non-compact spaces // Amer. J. Math. 1947. V. 69. № 2. P. 200-242.

[20] C. H. Dowker. Inductive dimension of completely normal spaces // Quart. J. Math. Oxford Ser. (2). 1953. V. 4. P. 267-281.

[21] C. H. Dowker. Local dimension of normal spaces // Quart. J. Math. Oxford. 1955. V. 6. № 22. P. 101-120.

[22] В.А. Дулев. О бесконечномерных $n$-многообразиях // Вестн. МГУ. Сер. 1. Матем., мех. 1996. № 5. С. $12-17$.

[23] В.И. Егоров. О метрической размерности точечных множеств // Докл. АН СССР. 1957. T. 112. № 5. C. $804-805$.

[24] В.И. Егоров. О метрической размерности точечных множеств // Матем. сб. 1959. T. 48. C. $227-250$.

[25] В.И. Егоров, Ю.М. Смирнов. О метрической размерности множеств // Труды 3-го Всесоюзного матем. съезда, Москва, 1956. Т. 4. М.: Изд-во АН СССР, 1959. С. 72.

[26] S. Eilenberg, E. Otto. Quelques propriétés caracteristiques de la dimension // Fund. Math. 1938. V. 31. P. 149-153.

[27] R. Engelking. Closed mappings on complete metric spaces // Fund. Math. 1971. V. 70. P. $103-107$.

[28] R. Engelking. Theory of dimension, finite and infinite. Lemgo: Heldermann, 1995. (Sigma Ser. Pure Math. V. 10.)

[29] В. В. Федорчук. Бикомпакты без промежуточных размерностей // Докл. АН СССР. 1973. T. 213. № 4. C. 795-797.

[30] V. V. Fedorchuk. Perfectly normal compact space without intermediate dimensions // Bull. Polish Acad. Sci. Math. 1975. V. 23. № 9. P. 975-979.

[31] В. В. Федорчук. Совместимость некоторых теорем общей топологии с аксиомами теории множеств // Докл. АН СССР. 1975. Т. 220. № 4. С. 786-788.

[32] В. В. Федорчук. Вполне замкнутые отображения и совместимость некоторых теорем общей топологии с аксиомами теории множеств // Матем. сб. 1976. Т. 99. № 1. С. 3-33.

[33] V. V. Fedorchuk. On the dimension of hereditarily normal spaces // Proc. London Math. Soc. (3). 1978. V. 36. №1. P. 163-175.

[34] В. В. Федорчук. Бесконечномерные бикомпакты // Изв. АН СССР. Сер. матем. 1978. T. 42. № 5. C. $1162-1178$.

[35] В. В. Федорчук. О размерности нигде не плотных подмножеств многообразий // Вестн. МГУ. Сер. 1. Матем., мех. 1992. № 2. С. 23-28.

[36] V. V. Fedorchuk. A differentiable manifold with noncoinciding dimensions // Topology Appl. 1993. V. 54. P. 221-239.

[37] В.В. Федорчук. Дифференцируемое многообразие с несовпадающими размерностями при СН // Матем. сб. 1995. Т. 186. № 1. С. 149-160. 
[38] В. В. Федорчук. О трансфинитной и когомологической размерности 4-многообразий // Труды МИАН. 1996. Т. 211. С. 193-212.

[39] В.В. Федорчук. Тождество Урысона и размерность многообразий // УМН. 1998. T. 53. № 5. C. $73-114$.

[40] В.В. Федорчук, М. Левин, Е. В. Щепин. О брауэровском определении размерности // УМН. 1999. Т. 54. № 2. С. 193-194.

[41] V.V. Fedorchuk, J. van Mill. Dimensiongrad for locally connected Polish spaces // Fund. Math. 2000. V. 163. №1. P. 77-82.

[42] В.В. Федорчук, В.В. Филиппов. Общая топология. Основные конструкции. М.: Изд-во МГУ, 1988.

[43] В. В. Федорчук, В. В. Филиппов. Многообразия с несовпадающими индуктивными размерностями // Матем. сб. 1992. Т. 183. №9. С. 22-44.

[44] В.В. Филиппов. О размерности нормалшных пространств // Докл. АН СССР. 1973. T. 209. № 4. C. $805-807$.

[45] Н. Хаджииванов. Продолжение отображений в сферы и проблема П. С. Александрова // Докл. АН СССР. 1970. Т. 194. № 3. С. 525-527.

[46] Н. Хаджииванов. О продолжении отображений в сферы и о счетных разлижениях тихоновских кубов // Матем. сб. 1971. Т. 84. № 1. С. 119-140.

[47] E. Hemmingsen. Some theorems on dimension theory for normal Hausdorff spaces // Duke Math. J. 1946. V. 13. P. 495-504.

[48] D.W. Henderson. An infinite-dimensional compactum with no positive-dimensional compact subsets. A simpler construction // Amer. J. Math. 1967. V. 89. P. 105-121.

[49] W. Hurewicz. Über unendlich-dimensionale Punktmengen // Proc. Roy. Acad. Amsterdam. 1928. V. 31. P. 916-922.

[50] W. Hurewicz, K. Menger. Dimension und Zusammenhangsstuffe // Math. Ann. 1928. V. 100. P. $618-633$.

[51] W. Hurewicz, H. Wallman. Dimension Theory. Princeton: Princeton Univ. Press, 1941.

[52] J. R. Isbell. On finite-dimensional uniform spaces // Pacific J. Math. 1956. V. 6. P. $107-121$.

[53] Д.Р. Исбелл. Об индуктивной размерности пространств близости // Докл. АН СССР. 1960. T. 134. № 1. C. 36-38.

[54] J. R. Isbell. On finite-dimensional uniform spaces. II // Pacific J. Math. 1962. V. 12. P. 291-302.

[55] J. R. Isbell. Uniform Spaces. Providence, RI: Amer. Math. Soc., 1964.

[56] Т. Йех. Теория множеств и метод форсинга. М.: Мир, 1973.

[57] R. B. Jensen. The fine structure of the constructible hierarchy // Ann. Math. Logic. 1972. V. 4. P. 229-308.

[58] А. В. Карасев. Об индуктивной размерности замкнутых подмножеств некоторых неметризуемых многообразий // Вестн. МГУ. Сер. 1. Матем., мех. 1997. № 5. С. 11-14.

[59] М. Катетов. О размерности метрических пространств // Докл. АН СССР. 1951. Т. 79. C. $189-191$.

[60] М. Катетов. О размерности несепарабельных пространств I // Czechoslovak Math. J. 1952. V. 2. P. 333-368.

[61] М. Катетов. О соотношении между метрической и топологической размерностью // Czechoslovak Math. J. 1958. V. 8. P. 163-166.

[62] G. Kozlowski, P. Zenor. A differentiale, perfectly normal, nonmetrizable manifold // Topology: Proc. Conf., Ohio Univ., 1979. V. 4. № 2, 1980. P. 453-461.

[63] А. Б. Куровский, Ю. М. Смирнов. О размерности Ird, определяемой с помощью ретракции // Czechoslovak Math. J. 1976. V. 26. № 1. P. 30-36.

[64] H. Lebesgue. Sur la non applicabilité de deux domaines appartenant respectivement à des espaces, de $n$ et $n+p$ dimensions // Math. Ann. 1911. V. 70. P. 166-168.

[65] H. Lebesgue. Sur les correspondences entre les points de deux espaces // Fund. Math. 1921. V. 2. P. 256-285. 
[66] Б.Т. Левшенко. О сильно-бесконечномерных пространствах // Вестн. МГУ. Сер. 1. Матем., мех. 1959. № 5. С. 219-228.

[67] Б. Т. Левшенко. О бесконечномерных пространствах // Докл. АН СССР. 1961. Т. 139. C. $286-289$.

[68] Б. Т. Левшенко. Пространства трансфиинитной размерности // Матем. сб. 1965. Т. 67. C. $225-266$.

[69] Б. Т. Левшенко. Размерность метрических пространств и ретракция // Fund. Math. 1969/70. V. 66. P. 1-5.

[70] Б. Т. Левшенко, Ю. М. Смирнов. Об одном свойстве нульмерных метрических пространств // Proceedings of the Second Prague Topol. Sympos., 1966, 1967. C. 241-242.

[71] J. van Mill, T.C. Przymusinski. There is no compactification theorem for the small inductive dimension // Topology Appl. 1982. V. 13. P. 133-136.

[72] K. Morita. On the dimension of normal spaces. I // Japan. J. Math. 1950. V. 20. P. 5-36.

[73] K. Morita. On the dimension of normal spaces. II // J. Math. Soc. Japan. 1950. V. 2. P. $16-33$.

[74] K. Morita. On dimension of product spaces // Amer. J. Math. 1953. V. 75. № 2. P. 205-223.

[75] K. Nagami. Monotone sequence of 0-dimensional subsets of metric spaces // Proc. Japan Acad. 1965. V. 41. P. 771-772.

[76] J. Nagata. On the countable sum of zero-dimensional spaces // Fund. Math. 1960. V. 48. P. 1-14.

[77] J. Nagata. On a universal $n$-dimensional set for metric spaces // J. Reine Angew. Math. 1960. V. 204. P. 132-138.

[78] J. Nagata. A remark on general imbedding theorems in dimension theory // Proc. Japan Acad. 1963. V. 39. P. 197-199.

[79] Г.М. Непомнящий, Ю. М. Смирнов. О ретракции отображений // Czechoslovak Math. J. 1979. V. 29. № 3. P. 366-377.

[80] В.А. Никифоров. Продолжение селекции к многозначному отображению и теорема двойственности Эйленберга-Борсука // Вестн. МГУ. Сер. 1. Матем., мех. 1987. №6 6 . C. $57-59$.

[81] P. Nyikos. The theory of nonmetrizable manifolds // Handbook of Set-Theoretic Topology. Amsterdam, 1984. P. 633-684.

[82] А. А. Одинцов. Змеевидные бикомпакты и некоторые вопросы теории размерности // Вестн. МГУ. Сер. 1. Матем., мех. 1986. № 1. С. 59-62.

[83] A. J. Ostaszewski. A perfectly normal countably compact scattered space which is not strongly zero-dimensional // J. London Math. Soc. (2). 1976. V. 14. № 1. P. 167-177.

[84] Б. А. Пасынков. Факторизационная теорема для незамкнутых множеств // Докл. АН CCCP. 1972. T. 202. C. 1274-1276.

[85] Б. А. Пасынков, В. В. Федорчук, В. В. Филиппов. Теория размерности // Итоги науки и техники. Алг. Топол. Геом. Т. 17. М.: ВИНИТИ, 1979. С. 229-306.

[86] H. Poincaré. L'espace et ses trois dimensions // Revue de Metaph. et de Morale. 1903. V. 11. P. 407-429.

[87] H. Poincaré. Pourquoi l'espace à trois dimensions // Revue de Metaph. et de Morale. 1912. V. 20. P. 483-504.

[88] E. Pol. A remark about Juhasz-Kunen-Rudin construction of a hereditarily separable non-Lindelöf space // Bull. Polish Acad. Sci. Math. 1976. V. 24. № 9. P. 749-751.

[89] E. Pol. A remark on countable-dimensional Cech-Stone bicompactifications // Manuscript, 1979.

[90] E. Pol. A remark concerning perfectly normal spaces with distinct local and global dimension // Topology Proc. 1991. V. 16. P. 125-132.

[91] E. Pol, R. Pol. A hereditarily normal strongly zero-dimensional space containing subspaces of arbitrary large dimension // General Topology and its Relations to Modern Analysis and Algebra IV: Proceedings of the Fourth Prague Topol. Symp., 1976, Part B. Prague, 1977. P. 357-360. 
[92] E. Pol, R. Pol. A hereditarily normal strongly zero-dimensional space with a subspace of positive dimension and an $N$-compact space of positive dimension // Fund. Math. 1977. V. 97. № 1. P. 43-50.

[93] R. Pol. On classification of weakly infinite-dimensional compacta // Fund. Math. 1983. V. 116. P. $169-188$.

[94] М. М. Постников. Гладкие многообразия. М.: Наука, 1987.

[95] P. Roy. Failure of equivalence of dimension concepts for metric spaces // Bull. Amer. Math. Soc. 1962. V. 68. P. 609-613.

[96] P. Roy. Nonequality of dimension for metric spaces // Trans. Amer. Math. Soc. 1968. V. 134. P. $117-132$.

[97] M. E. Rudin. The undecidability of the existence of a perfectly normal nonmetrizable manifold // Houston J. Math. 1979. V. 5. P. 249-252.

[98] Н. В. Савинов. Пример совершенно нормалшного бикомпакта без промежуточных размерностей // Вестн. МГУ. Сер. 1. Матем., мех. 1976. № 3. С. 52-56.

[99] A. W. Schurle. Compactification of strongly countable-dimensional spaces // Trans. Amer. Math. Soc. 1969. V. 136. P. 25-36.

[100] К. А. Ситников. Пример двумерного множества в трехмерном пространстве, допускающего сколь угодно малые деформации в одномерный полиэдр // Докл. АН СССР. 1953. T. 88 . C. $21-24$.

[101] К. А. Ситников. Комбинаторная топология незамкнутых множеств. II. Размерность // Матем. сб. 1955. Т. 37. С. 385-434.

[102] Е. Г. Скляренко. Бикомпактные расширения семибикомпактных пространств // Докл. AH CCCP. 1958. T. 120. №6. C. 1200-1203.

[103] Е. Г. Скляренко. О вложении нормальных пространств в бикомпакты того же веса и той же размерности // Докл. АН СССР. 1958. Т. 123. С. 36-39.

[104] Е. Г. Скляренко. Несколько замечаний о бесконечномерных пространствах // Докл. AH CCCP. 1959. T. 126. C. 1203-1206.

[105] Е. Г. Скляренко. О размерностных свойствах бесконечномерных пространств // Изв. АН CСCP. Сер. матем. 1959. Т. 23. С. 197-212.

[106] Ю. М. Смирнов. Некоторые соотношения в теории размерности // Матем. сб. 1951. T. 29. № 1. C. $157-172$.

[107] Ю. М. Смирнов. О нормалшно расположенных множествах нормальных пространств // Матем. сб. 1951. Т. 29. № 1. С. 173-176.

[108] Ю. М. Смирнов. О размерности пространств близости // Матем. сб. 1956. Т. 38. № 3. C. $283-302$.

[109] Ю. М. Смирнов. Геометрия бесконечных равномерных комплексов и $\delta$-размерность точечных множеств // Матем. сб. 1956. Т. 40. № 2. С. 137-156.

[110] Ю. М. Смирнов. О метрической размерности в смысле П. С. Александрова // Изв. АН СССР. Сер. матем. 1956. Т. 20. № 5. С. 679-684.

[111] Ю. М. Смирнов. Пример одномерного нормального пространства, не содержащегося ни в каком одномерном бикомпакте // Докл. АН СССР. 1957. Т. 117. № 6. С. 939-942.

[112] Ю. М. Смирнов. Пример вполне регулярного пространства с нормальным чеховским наростом, не обладающего свойством семибикомпактности // Докл. АН СССР. 1958. T. 120. №6. C. 1204-1206.

[113] Ю. М. Смирнов. Пример нульмерного нормального пространства, имеющего бесконечную размерность в смысле покрытий // Докл. АН СССР. 1958. Т. 123. № 1. С. 40-42.

[114] Ю. М. Смирнов. Теорема П. С. Александрова о существенных отображениях // Вестн. МГУ. Сер. 1. Матем., мех. 1959. № 5. С. 43-48.

[115] Ю. М. Смирнов. Об универсальных простраствах для некоторых классов бесконечномерных пространств // Изв. АН СССР. Сер. матем. 1959. Т. 23. № 2. С. 185-196.

[116] Yu. Smirnov. On dimensional properties of infinite-dimensional spaces // General Topology and its Relations to Modern Analysis and Algebra IV: Proc. Sympos., Prague, 1961, 1962. P. $334-336$. 
[117] Ю. М. Смирнов. Несколько замечаний о трансфинитной размерности // Докл. АН CССР. 1961. Т. 141. № 4. С. 814-817.

[118] Ю. М. Смирнов.О трансфинитной размерности // Матем. сб. 1962. Т. 58.№ 4. C. $415-422$.

[119] Yu. M. Smirnov. Einige Bemerkungen zu meinem Bericht "Über die Dimension der Adjunkten bei Kompaktifizierungen" // Monatsberichte Deutsch. Akad. Wiss. 1965. V. 7. № 10/11. P. $750-753$.

[120] Ю. М. Смирнов. О размерности наростов бикомпактных расширений близостных и топологических пространств // Матем. сб. 1966. Т. 69. № 1. С. 141-160.

[121] Ю. М. Смирнов. О размерности наростов близостных и топологических пространств // Докл. АН СССР. 1966. Т. 168. № 3. С. 528-531.

[122] Ю. М. Смирнов. О размерности наростов бикомпактных расширений близостных и топологических пространств. II // Матем. сб. 1966. Т. 71. № 4. С. 454-482.

[123] Ю. М. Смирнов. Уплотнения на бикомпакты и связь с бикомпактными расширениями и с ретракцией // Fund. Math. 1968. V. 63. № 2. P. 199-211.

[124] Ю. М. Смирнов, Е. Г. Скляренко. Некоторые вопросы теории размерности // Труды 4-го Всесоюзного математ. съезда, Ленинград, 1961. Т. І. Пленарные доклады. Л.: Изд-во АН СССР, 1963. С. 219-226.

[125] R. Solovay, S. Tennenbaum. Iterated Cohen extensions and Souslin's problem // Ann. of Math. (2). 1971. V. 94. P. 201-245.

[126] Z. Szentmiklossy. $S$-spaces and $L$-spaces under Martin's axiom // Colloq. Math. Soc. János Bolyai. 1980. V. 23. P. 1139-1145.

[127] L. A. Tumarkin. Sur la stucture dimensionnelle des ensembles fermes // C. R. Acad. Sci. Paris. 1928. V. 186. P. 420-422.

[128] P. Urysohn. Memoire sur les multiplicités cantoriennes // Fund. Math. 1925. V. 7. P. 30-137.

[129] P. Urysohn. Memoire sur les multiplicités cantoriennes (suite) // Fund. Math. 1926. V. 8. P. 225-359.

[130] Н. Б. Веденисов. Замечание о размерности топологических пространств // Уч. записки Моск. ун-та. 1939. Т. 30. С. 131-140.

[131] H. Wallman. Lattices and topological spaces // Ann. of Math. (2). 1938. V. 39. P. $112-126$.

[132] W. A. R. Weiss. Countably compact spaces and Martin's axiom // Canad. J. Math. 1978. V. 30. P. 243-249.

[133] А. В. Зарелуа. О теореме Гуревича // Докл. АН СССР. 1961. Т. 141. № 4. С. 777-780.

[134] А. В. Зарелуа. О теореме Гуревича // Матем. сб. 1963. Т. 60. № 1. С. 17-28.

[135] А. В. Зарелуа. О равенстве размерностей // Матем. сб. 1963. Т. 62. С. 295-319.

[136] А. В. Зарелуа. О продолжении отображений на расширения, обладающие некоторыми специальными свойствами // Сиб. матем. журн. 1964. Т. 5. С. 532-548.

[137] В. П. Золотарев. О размерности подпространств // Вестн. МГУ. Сер. 1. Матем., мех. 1975. № 5. C. 10-12. 